\title{
EFFECTIVE RESULTS FOR UNIT EQUATIONS OVER FINITELY GENERATED DOMAINS
}

\author{
JAN-HENDRIK EVERTSE AND KÁLMÁN GYŐRY
}

\begin{abstract}
Let $A \supset \mathbb{Z}$ be a commutative domain which is finitely generated over $\mathbb{Z}$ as a $\mathbb{Z}$-algebra and let $a, b, c$ be non-zero elements of $A$. Extending earlier work of Siegel [25, 1921], Mahler [18, 1933] and Parry [20, 1950], Lang [13, 1960] proved that the equation $\left(^{*}\right) a \varepsilon+b \eta=c$ in $\varepsilon, \eta \in A^{*}$ has only finitely many solutions. Using Baker's theory of logarithmic forms, Győry [6, 1974], [7, 1979] proved that the solutions of $(*)$ can be determined effectively if $A$ is contained in an algebraic number field. In this paper we prove, in a precise quantitative form, an effective finiteness result for equations $\left(^{*}\right)$ over an arbitrary domain $A$ of characteristic 0 which is finitely generated over $\mathbb{Z}$. Our main tools are already existing effective finiteness results for $(*)$ over number fields and function fields, an effective specialization argument of Győry [8, 1983], 9, 1984], and effective results of Seidenberg [24, 1974] and Aschenbrenner [1, 2004] on linear equations over polynomial rings. We prove also an effective result for the exponential equation $a \gamma_{1}^{v_{1}} \cdots \gamma_{s}^{v_{s}}+b \gamma_{1}^{w_{1}} \cdots \gamma_{s}^{w_{s}}=c$ in integers $v_{1}, \ldots, w_{s}$, where $a, b, c$ and $\gamma_{1}, \ldots, \gamma_{s}$ are non-zero elements of $A$.
\end{abstract}

\section{INTRODUCTION}

Let $A \supset \mathbb{Z}$ be a commutative domain which is finitely generated over $\mathbb{Z}$ as a $\mathbb{Z}$-algebra. As usual, we denote by $A^{*}$ the unit group of $A$. We consider equations

$$
a \varepsilon+b \eta=c \text { in } \varepsilon, \eta \in A^{*}
$$

where $a, b, c$ are non-zero elements of $A$. Such equations, usually called unit equations, have a great number of applications. For instance, the ring

2010 Mathematics Subject Classification: Primary 11D61; Secondary: 11J86.

Keywords and Phrases: Unit equations, finitely generated domains, effective finiteness results.

July 29, 2011. 
of $S$-integers in an algebraic number field is finitely generated over $\mathbb{Z}$, so the $S$-unit equation in two unknowns is a special case of (1.1). In this paper, we consider equations (1.1) in the general case, where $A$ may contain transcendental elements, too.

Siegel [25, 1921] proved that (1.1) has only finitely many solutions in the case that $A$ is the ring of integers of a number field, and Mahler [18, 1933] did this in the case that $A=\mathbb{Z}\left[1 / p_{1} \cdots p_{t}\right]$ for certain primes $p_{1}, \ldots, p_{t}$. For $S$-unit equations over number fields, the finiteness of the number of solutions of (1.1) follows from work of Parry [20, 1950]. Finally, Lang [13, 1960] proved for arbitrary finitely generated domains $A$ that (1.1) has only finitely many solutions. The proofs of all these results are ineffective.

Baker [2, 1968] and Coates [5, 1968/69] implicitly proved effective finiteness results for certain special $(S-)$ unit equations. Later, Győry [6, 1974], [7. 1979] showed, in the case that $A$ is the ring of $S$-integers in a number field, that the solutions of (1.1) can be determined effectively in principle. His proof is based on estimates for linear forms in ordinary and $p$-adic logarithms of algebraic numbers. In his papers [8, 1983] and [9, 1984], Győry introduced an effective specialization argument, and he used this to establish effective finiteness results for decomposable form equations and discriminant equations over a wide class of finitely generated domains $A$ containing both algebraic and transcendental elements, of which the elements have some "good" effective representations. His results contain as a special case an effective finiteness result for equations (1.1) over these domains. Győry's method of proof could not be extended to arbitrary finitely generated domains $A$.

It is the purpose of this paper to prove an effective finiteness result for (1.1) over arbitrary finitely generated domains $A$. In fact, we give a quantitative statement, with effective upper bounds for the "sizes" of the solutions $\varepsilon, \eta$. The main new ingredient of our proof is an effective result by Aschenbrenner [1, 2004] on systems of linear equations over polynomial rings over $\mathbb{Z}$.

We introduce the notation used in our theorems. Let again $A \supset \mathbb{Z}$ be a commutative domain which is finitely generated over $\mathbb{Z}$, say $A=$ $\mathbb{Z}\left[z_{1}, \ldots, z_{r}\right]$. Let $I$ be the ideal of polynomials $f \in \mathbb{Z}\left[X_{1}, \ldots, X_{r}\right]$ such 
that $f\left(z_{1}, \ldots, z_{r}\right)=0$. Then $I$ is finitely generated, hence

$$
A \cong \mathbb{Z}\left[X_{1}, \ldots, X_{r}\right] / I, \quad I=\left(f_{1}, \ldots, f_{m}\right)
$$

for some finite set of polynomials $f_{1}, \ldots, f_{m} \in \mathbb{Z}\left[X_{1}, \ldots, X_{r}\right]$. We observe here that given $f_{1}, \ldots, f_{m}$, it can be checked effectively whether $A$ is a domain containing $\mathbb{Z}$. Indeed, this holds if and only if $I$ is a prime ideal of $\mathbb{Z}\left[X_{1}, \ldots, X_{r}\right]$ with $I \cap \mathbb{Z}=(0)$, and the latter can be checked effectively for instance using Aschenbrenner [1, Prop. 4.10, Cor. 3.5].

Denote by $K$ the quotient field of $A$. For $\alpha \in A$, we call $f$ a representative for $\alpha$, or say that $f$ represents $\alpha$ if $f \in \mathbb{Z}\left[X_{1}, \ldots, X_{r}\right]$ and $\alpha=f\left(z_{1}, \ldots, z_{r}\right)$. Further, for $\alpha \in K$, we call $(f, g)$ a pair of representatives for $\alpha$ or say that $(f, g)$ represents $\alpha$ if $f, g \in \mathbb{Z}\left[X_{1}, \ldots, X_{r}\right], g \notin I$ and $\alpha=f\left(z_{1}, \ldots, z_{r}\right) / g\left(z_{1}, \ldots, z_{r}\right)$. We say that $\alpha \in A$ (resp. $\alpha \in K$ ) is given if a representative (resp. pair of representatives) for $\alpha$ is given.

To do explicit computations in $A$ and $K$, one needs an ideal membership algorithm for $\mathbb{Z}\left[X_{1}, \ldots, X_{r}\right]$, that is an algorithm which decides for any given polynomial and ideal of $\mathbb{Z}\left[X_{1}, \ldots, X_{r}\right]$ whether the polynomial belongs to the ideal. In the literature there are various such algorithms; we mention only the algorithm of Simmons [26, 1970], and the more precise algorithm of Aschenbrenner [1, 2004] which plays an important role in our paper; see Lemma 2.5 below for a statement of his result. One can perform arithmetic operations on $A$ and $K$ by using representatives. Further, one can decide effectively whether two polynomials $f_{1}, f_{2}$ represent the same element of $A$, i.e., $f_{1}-f_{2} \in I$, or whether two pairs of polynomials $\left(f_{1}, g_{1}\right),\left(f_{2}, g_{2}\right)$ represent the same element of $K$, i.e., $f_{1} g_{2}-f_{2} g_{1} \in I$, by using one of the ideal membership algorithms mentioned above.

The degree $\operatorname{deg} f$ of a polynomial $f \in \mathbb{Z}\left[X_{1}, \ldots, X_{r}\right]$ is by definition its total degree. By the logarithmic height $h(f)$ of $f$ we mean the logarithm of the maximum of the absolute values of its coefficients. The size of $f$ is defined by $s(f):=\max (1, \operatorname{deg} f, h(f))$. Clearly, there are only finitely many polynomials in $\mathbb{Z}\left[X_{1}, \ldots, X_{r}\right]$ of size below a given bound, and these can be determined effectively.

Theorem 1.1. Assume that $r \geq 1$. Let $\widetilde{a}, \widetilde{b}, \widetilde{c}$ be representatives for $a, b, c$, respectively. Assume that $f_{1}, \ldots, f_{m}$ and $\widetilde{a}, \widetilde{b}, \widetilde{c}$ all have degree at most $d$ and logarithmic height at most $h$, where $d \geq 1, h \geq 1$. Then for each solution $(\varepsilon, \eta)$ of (1.1), there are representatives $\widetilde{\varepsilon}, \widetilde{\varepsilon}, \widetilde{\eta}, \widetilde{\eta}^{\prime}$ of $\varepsilon, \varepsilon^{-1}, \eta, \eta^{-1}$, 
respectively, such that

$$
s(\widetilde{\varepsilon}), s(\widetilde{\varepsilon}), s(\widetilde{\eta}), s\left(\widetilde{\eta}^{\prime}\right) \leq \exp \left((2 d)^{c_{1}^{r}}(h+1)\right),
$$

where $c_{1}$ is an effectively computable absolute constant $>1$.

By a theorem of Roquette [22, 1958], the unit group of a domain finitely generated over $\mathbb{Z}$ is finitely generated. In the case that $A=O_{S}$ is the ring of $S$-integers of a number field it is possible to determine effectively a system of generators for $A^{*}$, and this was used by Györy in his effective finiteness proof for (1.1) with $A=O_{S}$. However, no general algorithm is known to determine a system of generators for the unit group of an arbitrary finitely generated domain $A$. In our proof of Theorem 1.1, we do not need any information on the generators of $A^{*}$.

By combining Theorem 1.1 with an ideal membership algorithm for $\mathbb{Z}\left[X_{1}, \ldots, X_{r}\right]$, one easily deduces the following:

Corollary 1.2. Given $f_{1}, \ldots, f_{m}, a, b, c$, the solutions of (1.1) can be determined effectively.

Proof. Clearly, $\varepsilon, \eta$ is a solution of (1.1) if and only if there are polynomials $\widetilde{\varepsilon}, \widetilde{\varepsilon}, \widetilde{\eta}, \widetilde{\eta}^{\prime} \in \mathbb{Z}\left[X_{1}, \ldots, X_{r}\right]$ such that $\widetilde{\varepsilon}, \widetilde{\eta}$ represent $\varepsilon, \eta$, and

$$
\widetilde{a} \cdot \widetilde{\varepsilon}+\widetilde{b} \cdot \widetilde{\eta}-\widetilde{c}, \widetilde{\varepsilon} \cdot \widetilde{\varepsilon}-1, \tilde{\eta} \cdot \widetilde{\eta}^{\prime}-1 \in I .
$$

Thus, we obtain all solutions of (1.1) by checking, for each quadruple of polynomials $\widetilde{\varepsilon}, \widetilde{\varepsilon}, \widetilde{\eta}, \widetilde{\eta}^{\prime} \in \mathbb{Z}\left[X_{1}, \ldots, X_{r}\right]$ of size at most $\exp \left((2 d)^{c_{1}^{r}}(h+1)\right)$ whether it satisfies (1.3). Further, using the ideal membership algorithm, it can be checked effectively whether two different pairs $(\widetilde{\varepsilon}, \widetilde{\eta})$ represent the same solution of (1.1). Thus, we can make a list of representatives, one for each solution of (1.1).

Let $\gamma_{1}, \ldots, \gamma_{s}$ be multiplicatively independent elements of $K^{*}$ (the multiplicative independence of $\gamma_{1}, \ldots, \gamma_{s}$ can be checked effectively for instance using Lemma 7.2 below). Let again $a, b, c$ be non-zero elements of $A$ and consider the equation

$$
a \gamma_{1}^{v_{1}} \cdots \gamma_{s}^{v_{s}}+b \gamma_{1}^{w_{1}} \cdots \gamma_{s}^{w_{s}}=c \text { in } v_{1}, \ldots, v_{s}, w_{1}, \ldots, w_{s} \in \mathbb{Z} .
$$

Theorem 1.3. Let $\widetilde{a}, \widetilde{b}, \widetilde{c}$ be representatives for $a, b, c$ and for $i=1, \ldots, s$, let $\left(g_{i 1}, g_{i 2}\right)$ be a pair of representatives for $\gamma_{i}$. Suppose that $f_{1}, \ldots, f_{m}$, 
$\widetilde{a}, \widetilde{b}, \widetilde{c}$, and $g_{i 1}, g_{i 2}(i=1, \ldots, s)$ all have degree at most $d$ and logarithmic height at most $h$, where $d \geq 1, h \geq 1$. Then for each solution $\left(v_{1}, \ldots, w_{s}\right)$ of (1.4) we have

$$
\max \left(\left|v_{1}\right|, \ldots,\left|v_{s}\right|,\left|w_{1}\right|, \ldots,\left|w_{s}\right|\right) \leq \exp \left((2 d)^{c_{2}^{r+s}}(h+1)\right),
$$

where $c_{2}$ is an effectively computable absolute constant $>1$.

An immediate consequence of Theorem 1.3 is that for given $f_{1}, \ldots, f_{m}, a, b, c$ and $\gamma_{1}, \ldots, \gamma_{s}$, the solutions of (1.4) can be determined effectively.

Since every domain finitely generated over $\mathbb{Z}$ has a finitely generated unit group, equation (1.1) maybe viewed as a special case of (1.4). But since no general effective algorithm is known to find a finite system of generators for the unit group of a finitely generated domain, we cannot deduce an effective result for (1.1) from Theorem 1.3. In fact, we argue reversely, and prove Theorem 1.3 by combining Theorem 1.1 with an effective result on Diophantine equations of the type $\gamma_{1}^{v_{1}} \cdots \gamma_{s}^{v_{s}}=\gamma_{0}$ in integers $v_{1}, \ldots, v_{s}$, where $\gamma_{1}, \ldots, \gamma_{s}, \gamma_{0} \in K^{*}$ (see Corollary 7.3 below).

The idea of the proof of Theorem 1.1 is roughly as follows. We first estimate the degrees of the representatives of $\varepsilon, \eta$ using Mason's effective result [19, 1983] on two term $S$-unit equations over function fields. Next, we apply many different specialization maps $A \rightarrow \overline{\mathbb{Q}}$ to (1.1) and obtain in this manner a large system of $S$-unit equations over number fields. By applying an existing effective finiteness result for such $S$-unit equations (e.g., Győry and Yu [10, 2006]) we collect enough information to retrieve an effective upper bound for the heights of the representatives of $\varepsilon, \eta$. In our proof, we apply the specialization maps on a domain $B \supset A$ of a special type which can be dealt with more easily. In the construction of $B$, we use an effective result of Seidenberg [24, 1974] on systems of linear equations over polynomial rings over arbitrary fields. To be able to go back to equation (1.1) over $A$, we need an effective procedure to decide whether a given element of $B$ belongs to $A^{*}$. For this decision procedure, we apply an effective result of Aschenbrenner [1, 2004] on systems of linear equations over polynomial rings over $\mathbb{Z}$.

The above approach was already followed by Győry [8, 1983], [9, 1984]. However, in these papers the domains $A$ are represented over $\mathbb{Z}$ in a different way. Hence, to select those solutions from $B$ of the equations under 
consideration which belong to $A$, certain restrictions on the domains $A$ had to be imposed.

In a forthcoming paper, we will give some applications of our above theorems and our method of proof to other classes of Diophantine equations over finitely generated domains.

\section{EfFective Linear Algebra OVER POLYNOMIAL RINGS}

We have collected some effective results for systems of linear equations to be solved in polynomials with coefficients in a field, or with coefficients in $\mathbb{Z}$.

Here and in the remainder of this paper, we write

$$
\log ^{*} x:=\max (1, \log x) \text { for } x>0, \log ^{*} 0:=1 .
$$

We use notation $O(\cdot)$ as an abbreviation for $c \times$ the expression between the parentheses, where $c$ is an effectively computable absolute constant. At each occurrence of $O(\cdot)$, the value of $c$ may be different.

Given a commutative domain $R$, we denote by $R^{m, n}$ the $R$-module of $m \times n$-matrices with entries in $R$ and by $R^{n}$ the $R$-module of $n$-dimensional column vectors with entries in $R$. Further, $\mathrm{GL}_{n}(R)$ denotes the group of matrices in $R^{n, n}$ with determinant in the unit group $R^{*}$. The degree of a polynomial $f \in R\left[X_{1}, \ldots, X_{N}\right]$, that is, its total degree, is denoted by $\operatorname{deg} f$.

From matrices $A, B$ with the same number of rows, we form a matrix $[A, B]$ by placing the columns of $B$ after those of $A$. Likewise, from two matrices $A, B$ with the same number of columns we form $\left[{ }_{B}^{A}\right]$ by placing the rows of $B$ below those of $A$.

The logarithmic height $h(S)$ of a finite set $S=\left\{a_{1}, \ldots, a_{t}\right\} \subset \mathbb{Z}$ is defined by $h(S):=\log \max \left(\left|a_{1}\right|, \ldots,\left|a_{t}\right|\right)$. The logarithmic height $h(U)$ of a matrix with entries in $\mathbb{Z}$ is defined by the logarithmic height of the set of entries of $U$. The logarithmic height $h(f)$ of a polynomial with coefficients in $\mathbb{Z}$ is the logarithmic height of the set of coefficients of $f$.

Lemma 2.1. Let $U \in \mathbb{Z}^{m, n}$. Then the $\mathbb{Q}$-vector space of $\mathbf{y} \in \mathbb{Q}^{n}$ with $U \mathbf{y}=\mathbf{0}$ is generated by vectors in $\mathbb{Z}^{n}$ of logarithmic height at most $m h(U)+$ $\frac{1}{2} m \log m$. 
Proof. Without loss of generality we may assume that $U$ has rank $m$, and moreover, that the matrix $B$ consisting of the first $m$ columns of $U$ is invertible. Let $\Delta:=\operatorname{det} B$. By multiplying with $\Delta B^{-1}$, we can rewrite $U \mathbf{y}=\mathbf{0}$ as $\left[\Delta I_{m}, C\right] \mathbf{y}=\mathbf{0}$, where $I_{m}$ is the $m \times m$-unit matrix, and $C$ consists of $m \times m$-subdeterminants of $U$. The solution space of this system is generated by the columns of $\left[\overline{\Delta I}_{n-m}^{-C}\right]$. An application of Hadamard's inequality gives the upper bound from the lemma for the logarithmic heights of these columns.

Proposition 2.2. Let $F$ be a field, $N \geq 1$, and $R:=F\left[X_{1}, \ldots, X_{N}\right]$. Further, let $A$ be an $m \times n$-matrix and $\mathbf{b}$ and $m$-dimensional column vector, both consisting of polynomials from $R$ of degree $\leq d$ where $d \geq 1$.

(i) The $R$-module of $\mathbf{x} \in R^{n}$ with $A \mathbf{x}=\mathbf{0}$ is generated by vectors $\mathbf{x}$ whose coordinates are polynomials of degree at most $(2 m d)^{2^{N}}$.

(ii) Suppose that $A \mathbf{x}=\mathbf{b}$ is solvable in $\mathbf{x} \in R^{n}$. Then it has a solution $\mathbf{x}$ whose coordinates are polynomials of degree at most $(2 m d)^{2^{N}}$.

Proof. See Aschenbrenner [1, Thms. 3.2, 3.4]. Results of this type were obtained earlier, but not with a completely correct proof, by Hermann [12, 1926] and Seidenberg [24, 1974].

Corollary 2.3. Let $R:=\mathbb{Q}\left[X_{1}, \ldots, X_{N}\right]$. Further, Let $A$ be an $m \times n$-matrix of polynomials in $\mathbb{Z}\left[X_{1}, \ldots, X_{N}\right]$ of degrees at most $d$ and logarithmic heights at most $h$ where $d \geq 1, h \geq 1$. Then the $R$-module of $\mathbf{x} \in R^{n}$ with $A \mathbf{x}=\mathbf{0}$ is generated by vectors $\mathbf{x}$, consisting of polynomials in $\mathbb{Z}\left[X_{1}, \ldots, X_{N}\right]$ of degree at most $(2 m d)^{2^{N}}$ and height at most $(2 m d)^{6^{N}}(h+1)$.

Proof. By Proposition 2.2 (i) we have to study $A \mathbf{x}=\mathbf{0}$, restricted to vectors $\mathbf{x} \in R^{n}$ consisting of polynomials of degree at most $(2 d)^{2^{N}}$. The set of these $\mathbf{x}$ is a finite dimensional $\mathbb{Q}$-vector space, and we have to prove that it is generated by vectors whose coordinates are polynomials in $\mathbb{Z}\left[X_{1}, \ldots, X_{N}\right]$ of logarithmic height at most $(2 m d)^{6^{N}}(h+1)$.

If $\mathbf{x}$ consists of polynomials of degree at most $(2 m d)^{2^{N}}$, then $A \mathbf{x}$ consists of $m$ polynomials with coefficients in $\mathbb{Q}$ of degrees at most $(2 m d)^{2^{N}}+d$, all whose coefficients have to be set to 0 . This leads to a system of linear equations $U \mathbf{y}=\mathbf{0}$, where $\mathbf{y}$ consists of the coefficients of the polynomials in $\mathbf{x}$ and $U$ consists of integers of logarithmic heights at most $h$. Notice 
that the number $m^{*}$ of rows of $U$ is $m$ times the number of monomials in $N$ variables of degree at most $(2 m d)^{2^{N}}+d$, that is

$$
m^{*} \leq m\left(\begin{array}{c}
(2 m d)^{2^{N}}+d+N \\
N
\end{array}\right) .
$$

By Lemma 2.1 the solution space of $U \mathbf{y}=\mathbf{0}$ is generated by integer vectors of logarithmic height at most

$$
m^{*} h+\frac{1}{2} m^{*} \log m^{*} \leq(2 m d)^{6^{N}}(h+1) .
$$

This completes the proof of our corollary.

Lemma 2.4. Let $U \in \mathbb{Z}^{m, n}, \mathbf{b} \in \mathbb{Z}^{m}$ be such that $U \mathbf{y}=\mathbf{b}$ is solvable in $\mathbb{Z}^{n}$. Then it has a solution $\mathbf{y} \in \mathbb{Z}^{n}$ with $h(\mathbf{y}) \leq m h([U, \mathbf{b}])+\frac{1}{2} m \log m$.

Proof. Assume without loss of generality that $U$ and $[U, \mathbf{b}]$ have rank $m$. By a result of Borosh, Flahive, Rubin and Treybig [4, 1989], $U \mathbf{y}=\mathbf{b}$ has a solution $\mathbf{y} \in \mathbb{Z}^{n}$ such that the absolute values of the entries of $\mathbf{y}$ are bounded above by the maximum of the absolute values of the $m \times m$-subdeterminants of $[U, \mathbf{b}]$. The upper bound for $h(\mathbf{y})$ as in the lemma easily follows from Hadamard's inequality.

Proposition 2.5. Let $N \geq 1$ and let $f_{1}, \ldots, f_{m}, b \in \mathbb{Z}\left[X_{1}, \ldots, X_{N}\right]$ be polynomials of degrees at most $d$ and logarithmic heights at most $h$ where $d \geq 1$, $h \geq 1$, such that

$$
f_{1} x_{1}+\cdots+f_{m} x_{m}=b
$$

is solvable in $x_{1}, \ldots, x_{m} \in \mathbb{Z}\left[X_{1}, \ldots, x_{N}\right]$. Then (2.1) has a solution in polynomials $x_{1}, \ldots, x_{m} \in \mathbb{Z}\left[X_{1}, \ldots, X_{N}\right]$ with

for $i=1, \ldots, m$.

Proof. Aschenbrenner's main theorem [1, Theorem A] states that Eq. (2.1) has a solution $x_{1}, \ldots, x_{m} \in \mathbb{Z}\left[X_{1}, \ldots, X_{N}\right]$ with $\operatorname{deg} x_{i} \leq d_{0}$ for $i=1, \ldots, m$, where

$$
d_{0}=(2 d)^{\exp O\left(N \log ^{*} N\right)}(h+1) .
$$

So it remains to show the existence of a solution with small logarithmic height. 
Let us restrict to solutions $\left(x_{1}, \ldots, x_{m}\right)$ of (2.1) of degree $\leq d_{0}$, and denote by $\mathbf{y}$ the vector of coefficients of the polynomials $x_{1}, \ldots, x_{m}$. Then (2.1) translates into a system of linear equations $U \mathbf{y}=\mathbf{b}$ which is solvable over $\mathbb{Z}$. Here, the number of equations, i.e., number of rows of $U$, is equal to $m^{*}:=\left(\begin{array}{c}d_{0}+d+N \\ N\end{array}\right)$. Further, $h(U, \mathbf{b}) \leq h$. By Lemma 2.4, $U \mathbf{y}=\mathbf{b}$ has a solution $\mathbf{y}$ with coordinates in $\mathbb{Z}$ of height at most

$$
m^{*} h+\frac{1}{2} m^{*} \log m^{*} \leq(2 d)^{\exp O\left(N \log ^{*} N\right)}(h+1)^{N+1} .
$$

It follows that (2.1) has a solution $x_{1}, \ldots, x_{m} \in \mathbb{Z}\left[X_{1}, \ldots, X_{N}\right]$ satisfying (2.2).

Remarks. 1. Aschenbrenner gives in [1] an example which shows that the upper bound for the degrees of the $x_{i}$ cannot depend on $d$ and $N$ only.

2. The above lemma gives an effective criterion for ideal membership in $\mathbb{Z}\left[X_{1}, \ldots, X_{N}\right]$. Let $b \in \mathbb{Z}\left[X_{1}, \ldots, X_{N}\right]$ be given. Further, suppose that an ideal $I$ of $\mathbb{Z}\left[X_{1}, \ldots, X_{N}\right]$ is given by a finite set of generators $f_{1}, \ldots, f_{m}$. By the above lemma, if $b \in I$ then there are $x_{1}, \ldots, x_{m} \in \mathbb{Z}\left[X_{1}, \ldots, X_{N}\right]$ with upper bounds for the degrees and heights as in (2.2) such that $b=\sum_{i=1}^{m} x_{i} f_{i}$. It requires only a finite computation to check whether such $x_{i}$ exist.

\section{A REDUCTION}

We reduce the general unit equation (1.1) to a unit equation over a domain $B$ of a special type which can be dealt with more easily.

Let again $A=\mathbb{Z}\left[z_{1}, \ldots, z_{r}\right] \supset \mathbb{Z}$ be a commutative domain finitely generated over $\mathbb{Z}$ and denote by $K$ the quotient field of $A$. We assume that $r>0$. We have

$$
A \cong \mathbb{Z}\left[X_{1}, \ldots, X_{r}\right] / I
$$

where $I$ is the ideal of polynomials $f \in \mathbb{Z}\left[X_{1}, \ldots, X_{r}\right]$ such that $f\left(z_{1}, \ldots, z_{r}\right)$ $=0$. The ideal $I$ is finitely generated. Let $d \geq 1, h \geq 1$ and assume that

$$
I=\left(f_{1}, \ldots, f_{m}\right) \text { with } \operatorname{deg} f_{i} \leq d, \quad h\left(f_{i}\right) \leq h(i=1, \ldots, m) .
$$

Suppose that $K$ has transcendence degree $q \geq 0$. In case that $q>0$, we assume without loss of generality that $z_{1}, \ldots, z_{q}$ form a transcendence basis of $K / \mathbb{Q}$. We write $t:=r-q$ and rename $z_{q+1}, \ldots, z_{r}$ as $y_{1}, \ldots, y_{t}$, 
respectively. In case that $t=0$ we have $A=\mathbb{Z}\left[z_{1}, \ldots, z_{q}\right], A^{*}=\{ \pm 1\}$ and Theorem 1.1 is trivial. So we assume henceforth that $t>0$.

Define

$$
\begin{aligned}
& A_{0}:=\mathbb{Z}\left[z_{1}, \ldots, z_{q}\right], \quad K_{0}:=\mathbb{Q}\left(z_{1}, \ldots, z_{q}\right) \text { if } q>0 \\
& A_{0}:=\mathbb{Z}, \quad K_{0}:=\mathbb{Q} \text { if } q=0 .
\end{aligned}
$$

Then

$$
A=A_{0}\left[y_{1}, \ldots, y_{t}\right], \quad K=K_{0}\left(y_{1}, \ldots, y_{t}\right) .
$$

Clearly, $K$ is a finite extension of $K_{0}$, so in particular an algebraic number field if $q=0$. Using standard algebra techniques, one can show that there exist $y \in A, f \in A_{0}$ such that $K=K_{0}(y), y$ is integral over $A_{0}$, and

$$
A \subseteq B:=A_{0}\left[f^{-1}, y\right], \quad a, b, c \in B^{*} .
$$

If $\varepsilon, \eta \in A^{*}$ is a solution to (1.1), then $\varepsilon_{1}:=a \varepsilon / c, \eta_{1}:=b \eta / c$ satisfy

$$
\varepsilon_{1}+\eta_{1}=1, \quad \varepsilon_{1}, \eta_{1} \in B^{*}
$$

At the end of this section, we formulate Proposition 3.8 which gives an effective result for equations of the type (3.3). More precisely, we introduce an other type of degree and height $\overline{\operatorname{deg}}(\alpha)$ and $\bar{h}(\alpha)$ for elements $\alpha$ of $B$, and give effective upper bounds for the $\overline{\operatorname{deg}}$ and $\bar{h}$ of $\varepsilon_{1}, \eta_{1}$. Subsequently we deduce Theorem 1.1.

The deduction of Theorem 1.1 is based on some auxiliary results which are proved first. We start with an explicit construction of $y, f$, with effective upper bounds in terms of $r, d, h$ and $a, b, c$ for the degrees and logarithmic heights of $f$ and of the coefficients in $A_{0}$ of the monic minimal polynomial of $y$ over $A_{0}$. Here we follow more or less Seidenberg [24, 1974]. Second, for a given solution $\varepsilon, \eta$ of (1.1), we derive effective upper bounds for the degrees and logarithmic heights of representatives for $\varepsilon, \varepsilon^{-1}, \eta, \eta^{-1}$ in terms of $\overline{\operatorname{deg}}\left(\varepsilon_{1}\right), \bar{h}\left(\varepsilon_{1}\right), \overline{\operatorname{deg}}\left(\eta_{1}\right), \bar{h}\left(\eta_{1}\right)$. Here we use Proposition 2.5 (Aschenbrenner's result).

We introduce some further notation. First let $q>0$. Then since $z_{1}, \ldots, z_{q}$ are algebraically independent, we may view them as independent variables, and for $\alpha \in A_{0}$, we denote by $\operatorname{deg} \alpha, h(\alpha)$ the total degree and logarithmic height of $\alpha$, viewed as polynomial in $z_{1}, \ldots, z_{q}$. In case that $q=0$, we have $A_{0}=\mathbb{Z}$, and we agree that $\operatorname{deg} \alpha=0, h(\alpha)=\log |\alpha|$ for $\alpha \in A_{0}$. We frequently use the following estimate, valid for all $q \geq 0$ : 
Lemma 3.1. Let $g_{1}, \ldots, g_{n} \in A_{0}$ and $g=g_{1} \cdots g_{n}$. Then

$$
\left|h(g)-\sum_{i=1}^{n} h\left(g_{i}\right)\right| \leq q \operatorname{deg} g \text {. }
$$

Proof. See Bombieri and Gubler [3, Lemma 1.6.11, pp. 27].

We write $\mathbf{Y}=\left(X_{q+1}, \ldots, X_{r}\right)$ and $K_{0}(\mathbf{Y}):=K_{0}\left(X_{q+1}, \ldots, X_{r}\right)$, etc. Given $f \in \mathbb{Q}\left(X_{1}, \ldots, X_{r}\right)$ we denote by $f^{*}$ the rational function of $K_{0}(\mathbf{Y})$ obtained by substituting $z_{i}$ for $X_{i}$ for $i=1, \ldots, q$ (and $f^{*}=f$ if $q=0$ ). We view elements $f^{*} \in A_{0}[\mathbf{Y}]$ as polynomials in $\mathbf{Y}$ with coefficients in $A_{0}$. We denote by $\operatorname{deg}_{\mathbf{Y}} f^{*}$ the (total) degree of $f^{*} \in K_{0}[\mathbf{Y}]$ with respect to $\mathbf{Y}$. We recall that $\operatorname{deg} g$ is defined for elements of $A_{0}$ and is taken with respect to $z_{1}, \ldots, z_{q}$. With this notation, we can rewrite (3.1), (3.2) as

$$
\left\{\begin{array}{l}
A \cong A_{0}[\mathbf{Y}] /\left(f_{1}^{*}, \ldots, f_{m}^{*}\right), \\
\operatorname{deg}_{\mathbf{Y}} f_{i}^{*} \leq d \text { for } i=1, \ldots, m \\
\text { the coefficients of } f_{1}^{*}, \ldots, f_{m}^{*} \text { in } A_{0} \text { have degrees at most } d \\
\text { and logarithmic heights at most } h .
\end{array}\right.
$$

Put $D:=\left[K: K_{0}\right]$ and denote by $\sigma_{1}, \ldots, \sigma_{D}$ the $K_{0}$ - isomorphic embeddings of $K$ in an algebraic closure $\overline{K_{0}}$ of $K_{0}$.

Lemma 3.2. (i) We have $D \leq d^{t}$.

(ii) There exist integers $a_{1}, \ldots, a_{t}$ with $\left|a_{i}\right| \leq D^{2}$ for $i=1, \ldots, t$ such that for $w:=a_{1} y_{1}+\cdots+a_{t} y_{t}$ we have $K=K_{0}(w)$.

Proof. (i) The set

$$
\mathcal{W}:=\left\{\mathbf{y} \in{\overline{K_{0}}}^{t}: f_{1}^{*}(\mathbf{y})=\cdots=f_{m}^{*}(\mathbf{y})=0\right\}
$$

consists precisely of the images of $\left(y_{1}, \ldots, y_{t}\right)$ under $\sigma_{1}, \ldots, \sigma_{D}$. So we have to prove that $\mathcal{W}$ has cardinality at most $d^{t}$.

In fact, this follows from a repeated application of Bézout's Theorem. Given $g_{1}, \ldots, g_{k} \in K_{0}[\mathbf{Y}]$, we denote by $\mathcal{V}\left(g_{1}, \ldots, g_{k}\right)$ the common set of zeros of $g_{1}, \ldots, g_{k}$ in ${\overline{K_{0}}}^{t}$. Let $g_{1}:=f_{1}^{*}$. Then by the version of Bézout's Theorem in Hartshorne [11, p. 53, Thm. 7.7], the irreducible components of $\mathcal{V}\left(g_{1}\right)$ have dimension $t-1$, and the sum of their degrees is at most $\operatorname{deg}_{\mathbf{Y}} g_{1} \leq d$. Take a $\overline{K_{0}}$-linear combination $g_{2}$ of $f_{1}^{*}, \ldots, f_{m}^{*}$ not vanishing identically on any of the irreducible components of $\mathcal{V}\left(g_{1}\right)$. For any of 
these components, say $\mathcal{V}$, the intersection of $\mathcal{V}$ and $\mathcal{V}\left(g_{2}\right)$ is a union of irreducible components, each of dimension $t-2$, whose degrees have sum at most $\operatorname{deg}_{\mathbf{Y}} g_{2} \cdot \operatorname{deg} \mathcal{V} \leq d \operatorname{deg} \mathcal{V}$. It follows that the irreducible components of $\mathcal{V}\left(g_{1}, g_{2}\right)$ have dimension $t-2$ and that the sum of their degrees is at most $d^{2}$. Continuing like this, we see that there are linear combinations $g_{1}, \ldots, g_{t}$ of $f_{1}^{*}, \ldots, f_{m}^{*}$ such that for $i=1, \ldots, t$, the irreducible components of $\mathcal{V}\left(g_{1}, \ldots, g_{i}\right)$ have dimension $d-i$ and the sum of their degrees is at most $d^{i}$. For $i=t$ it follows that $\mathcal{V}\left(g_{1}, \ldots, g_{t}\right)$ is a set of at most $d^{t}$ points. Since $\mathcal{W} \subseteq \mathcal{V}\left(g_{1}, \ldots, g_{t}\right)$ this proves $(\mathrm{i})$

(ii) Let $a_{1}, \ldots, a_{t}$ be integers. Then $w:=\sum_{i=1}^{t} a_{i} y_{i}$ generates $K$ over $K_{0}$ if and only if $\sum_{j=1}^{t} a_{j} \sigma_{i}\left(y_{j}\right)(i=1, \ldots, D)$ are distinct. There are integers $a_{i}$ with $\left|a_{i}\right| \leq D^{2}$ for which this holds.

Lemma 3.3. There are $\mathcal{G}_{0}, \ldots, \mathcal{G}_{D} \in A_{0}$ such that

$$
\begin{aligned}
& \sum_{i=0}^{D} \mathcal{G}_{i} w^{D-i}=0, \quad \mathcal{G}_{0} \mathcal{G}_{D} \neq 0, \\
& \operatorname{deg} \mathcal{G}_{i} \leq(2 d)^{\exp O(r)}, \quad h\left(\mathcal{G}_{i}\right) \leq(2 d)^{\exp O(r)}(h+1) \quad(i=0, \ldots, D) .
\end{aligned}
$$

Proof. In what follows we write $\mathbf{Y}=\left(X_{q+1}, \ldots, X_{r}\right)$ and $\mathbf{Y}^{\mathbf{u}}:=X_{q+1}^{u_{1}} \cdots X_{q+t}^{u_{t}}$, $|\mathbf{u}|:=u_{1}+\cdots+u_{t}$ for tuples of non-negative integers $\mathbf{u}=\left(u_{1}, \ldots, u_{t}\right)$. Further, we define $W:=\sum_{j=1}^{t} a_{j} X_{q+j}$.

$\mathcal{G}_{0}, \ldots, \mathcal{G}_{D}$ as in (3.5) clearly exist since $w$ has degree $D$ over $K_{0}$. By (3.4), there are $g_{1}^{*}, \ldots, g_{m}^{*} \in A_{0}[\mathbf{Y}]$ such that

$$
\sum_{i=0}^{D} \mathcal{G}_{i} W^{D-i}=\sum_{j=1}^{m} g_{j}^{*} f_{j}^{*}
$$

By Proposition 2.2 (ii), applied with the field $F=K_{0}$, there are polynomials $g_{j}^{*} \in K_{0}[\mathbf{Y}]$ (so with coefficients being rational functions in $\mathbf{z}$ ) satisfying (3.7) of degree at most $(2 \max (d, D))^{2^{t}} \leq\left(2 d^{t}\right)^{2^{t}}=: d_{0}$ in $\mathbf{Y}$. By multiplying $\mathcal{G}_{0}, \ldots, \mathcal{G}_{D}$ with an appropriate non-zero factor from $A_{0}$ we may assume that the $g_{j}^{*}$ are polynomials in $A_{0}[\mathbf{Y}]$ of degree at most $d_{0}$ in $\mathbf{Y}$. By considering (3.7) with such polynomials $g_{j}^{*}$, we obtain

$$
\sum_{i=0}^{D} \mathcal{G}_{i} W^{D-i}=\sum_{j=1}^{m}\left(\sum_{|\mathbf{u}| \leq d_{0}} g_{j, \mathbf{u}} \mathbf{Y}^{\mathbf{u}}\right) \cdot\left(\sum_{|\mathbf{v}| \leq d} f_{j, \mathbf{v}} \mathbf{Y}^{\mathbf{v}}\right)
$$


where $g_{j, \mathbf{u}} \in A_{0}$ and $f_{j}^{*}=\sum_{|\mathbf{v}| \leq d} f_{j, \mathbf{v}} \mathbf{Y}^{\mathbf{v}}$ with $f_{j, \mathbf{v}} \in A_{0}$. We view $\mathcal{G}_{0}, \ldots, \mathcal{G}_{D}$ and the polynomials $g_{j, \mathbf{u}}$ as the unknowns of (3.8). Then (3.8) has solutions with $\mathcal{G}_{0} \mathcal{G}_{D} \neq 0$.

We may view (3.8) as a system of linear equations $\mathcal{A} \mathbf{x}=\mathbf{0}$ over $K_{0}$, where $\mathbf{x}$ consists of $\mathcal{G}_{i}(i=0, \ldots, D)$ and $g_{j, \mathbf{u}}\left(j=1, \ldots, m,|\mathbf{u}| \leq d_{0}\right)$. By Lemma 3.2 and an elementary estimate, the polynomial $W^{D-i}=\left(\sum_{k=1}^{t} a_{k} X_{q+k}\right)^{D-i}$ has logarithmic height at most $O\left(D \log \left(2 D^{2} t\right)\right) \leq(2 d)^{O(t)}$. By combining this with (3.4), it follows that the entries of the matrix $\mathcal{A}$ are elements of $A_{0}$ of degrees at most $d$ and logarithmic heights at most $h_{0}:=\max \left((2 d)^{O(t)}, h\right)$. Further, the number of rows of $\mathcal{A}$ is at most the number of monomials in $\mathbf{Y}$ of degree at most $d_{0}+d$ which is bounded above by $m_{0}:=\left(\begin{array}{c}d_{0}+d+t \\ t\end{array}\right)$. So by Corollary 2.3, the solution module of (3.8) is generated by vectors $\mathbf{x}=\left(\mathcal{G}_{0}, \ldots, \mathcal{G}_{D},\left\{g_{i, \mathbf{u}}\right\}\right)$, consisting of elements from $A_{0}$ of degree and height at most

$$
\left(2 m_{0} d\right)^{2^{q}} \leq(2 d)^{\exp O(r)}, \quad\left(2 m_{0} d\right)^{6^{q}}\left(h_{0}+1\right) \leq(2 d)^{\exp O(r)}(h+1),
$$

respectively.

At least one of these vectors $\mathbf{x}$ must have $\mathcal{G}_{0} \mathcal{G}_{D} \neq 0$ since otherwise (3.8) would have no solution with $\mathcal{G}_{0} \mathcal{G}_{D} \neq 0$, contradicting (3.5). Thus, there exists a solution $\mathbf{x}$ whose components $\mathcal{G}_{0}, \ldots, \mathcal{G}_{D}$ satisfy both (3.5), (3.6). This proves our lemma.

It will be more convenient to work with

$$
y:=\mathcal{G}_{0} w=\mathcal{G}_{0} \cdot\left(a_{1} y_{1}+\cdots+a_{t} y_{t}\right) .
$$

In the case $D=1$ we set $y:=1$. The following properties of $y$ follow at once from Lemmas 3.13 .3 .

Corollary 3.4. We have $K=K_{0}(y), y \in A$, $y$ is integral over $A_{0}$, and $y$ has minimal polynomial $\mathcal{F}(X)=X^{D}+\mathcal{F}_{1} X^{D-1}+\cdots+\mathcal{F}_{D}$ over $K_{0}$ with

$$
\mathcal{F}_{i} \in A_{0}, \quad \operatorname{deg} \mathcal{F}_{i} \leq(2 d)^{\exp O(r)}, h\left(\mathcal{F}_{i}\right) \leq(2 d)^{\exp O(r)}(h+1)
$$

for $i=1, \ldots, D$.

Recall that $A_{0}=\mathbb{Z}$ if $q=0$ and $\mathbb{Z}\left[z_{1}, \ldots, z_{q}\right]$ if $q>0$, where in the latter case, $z_{1}, \ldots, z_{q}$ are algebraically independent. Hence $A_{0}$ is a unique factorization domain, and so the gcd of a finite set of elements of $A_{0}$ is welldefined and up to sign uniquely determined. With every element $\alpha \in K$ we 
can associate an up to sign unique tuple $P_{\alpha, 0}, \ldots, P_{\alpha, D-1}, Q_{\alpha}$ of elements of $A_{0}$ such that

$$
\alpha=Q_{\alpha}^{-1} \sum_{j=0}^{D-1} P_{\alpha, j} y^{j} \text { with } Q_{\alpha} \neq 0, \operatorname{gcd}\left(P_{\alpha, 0}, \ldots, P_{\alpha, D-1}, Q_{\alpha}\right)=1 .
$$

Put

$$
\left\{\begin{array}{l}
\overline{\operatorname{deg}} \alpha:=\max \left(\operatorname{deg} P_{\alpha, 0}, \ldots, \operatorname{deg} P_{\alpha, D-1}, \operatorname{deg} Q_{\alpha}\right), \\
\bar{h}(\alpha):=\max \left(h\left(P_{\alpha, 0}\right), \ldots, h\left(P_{\alpha, D-1}\right), h\left(Q_{\alpha}\right)\right)
\end{array} .\right.
$$

Then for $q=0$ we have $\overline{\operatorname{deg}} \alpha=0, \bar{h}(\alpha)=\log \max \left(\left|P_{\alpha, 0}\right|, \ldots,\left|P_{\alpha, D-1}\right|,\left|Q_{\alpha}\right|\right)$.

Lemma 3.5. Let $\alpha \in K^{*}$ and let $(a, b)$ be a pair of representatives for $\alpha$, with $a, b \in \mathbb{Z}\left[X_{1}, \ldots, X_{r}\right], b \notin I$. Put $d^{*}:=\max (d, \operatorname{deg} a, \operatorname{deg} b), h^{*}:=$ $\max (h, h(a), h(b))$. Then

$$
\overline{\operatorname{deg}} \alpha \leq\left(2 d^{*}\right)^{\exp O(r)}, \quad \bar{h}(\alpha) \leq\left(2 d^{*}\right)^{\exp O(r)}\left(h^{*}+1\right) .
$$

Proof. Consider the linear equation

$$
Q \cdot \alpha=\sum_{j=0}^{D-1} P_{j} y^{j}
$$

in unknowns $P_{0}, \ldots, P_{D-1}, Q \in A_{0}$. This equation has a solution with $Q \neq$ 0 , since $\alpha \in K=K_{0}(y)$ and $y$ has degree $D$ over $K_{0}$. Write again $\mathbf{Y}=$ $\left(X_{q+1}, \ldots, X_{r}\right)$ and put $Y:=\mathcal{G}_{0} \cdot\left(\sum_{j=1}^{t} a_{j} X_{q+j}\right)$. Let $a^{*}, b^{*} \in A_{0}[\mathbf{Y}]$ be obtained from $a, b$ by substituting $z_{i}$ for $X_{i}$ for $i=1, \ldots, q\left(a^{*}=a, b^{*}=b\right.$ if $q=0$ ). By (3.4), there are $g_{j}^{*} \in A_{0}[\mathbf{Y}]$ such that

$$
Q \cdot a^{*}-b^{*} \sum_{j=0}^{D-1} P_{j} Y^{j}=\sum_{j=1}^{m} g_{j}^{*} f_{j}^{*}
$$

By Proposition 2.2 (ii) this identity holds with polynomials $g_{j}^{*} \in A_{0}[\mathbf{Y}]$ of degree in $\mathbf{Y}$ at most $\left(2 \max \left(d^{*}, D\right)\right)^{2^{t}} \leq\left(2 d^{*}\right)^{t 2^{t}}$, where possibly we have to multiply $\left(P_{0}, \ldots, P_{D-1}, Q\right)$ with a non-zero element from $A_{0}$. Now completely similarly as in the proof of Lemma 3.3, one can rewrite (3.13) as a system of linear equations over $K_{0}$ and then apply Corollary 2.3. It follows that (3.12) is satisfied by $P_{0}, \ldots, P_{D-1}, Q \in A_{0}$ with $Q \neq 0$ and

$$
\begin{aligned}
& \operatorname{deg} P_{i}, \operatorname{deg} Q \leq\left(2 d^{*}\right)^{\exp O(r)}, \\
& h\left(P_{i}\right), h(Q) \leq\left(2 d^{*}\right)^{\exp O(r)}\left(h^{*}+1\right) \quad(i=0, \ldots, D-1) .
\end{aligned}
$$


By dividing $P_{0}, \ldots, P_{D-1}, Q$ by their gcd and using Lemma 3.1 we obtain $P_{\alpha, 0}, \ldots, P_{D-1, \alpha}, Q_{\alpha} \in A_{0}$ satisfying both (3.9) and

$$
\begin{aligned}
& \operatorname{deg} P_{i, \alpha}, \operatorname{deg} Q_{\alpha} \leq\left(2 d^{*}\right)^{\exp O(r)} \\
& h\left(P_{i, \alpha}\right), h\left(Q_{\alpha}\right) \leq\left(2 d^{*}\right)^{\exp O(r)}\left(h^{*}+1\right) \quad(i=0, \ldots, D-1) .
\end{aligned}
$$

Lemma 3.6. Let $\alpha_{1}, \ldots, \alpha_{n} \in K^{*}$. For $i=1, \ldots, n$, let $\left(a_{i}, b_{i}\right)$ be a pair of representatives for $\alpha_{i}$, with $a_{i}, b_{i} \in \mathbb{Z}\left[X_{1}, \ldots, X_{r}\right], b_{i} \notin I$. Put

$$
\begin{aligned}
d^{* *} & :=\max \left(d, \operatorname{deg} a_{1}, \operatorname{deg} b_{1}, \ldots, \operatorname{deg} a_{n}, \operatorname{deg} b_{n}\right), \\
h^{* *} & :=\max \left(h, h\left(a_{1}\right), h\left(b_{1}\right), \ldots, h\left(a_{n}\right), h\left(b_{n}\right)\right) .
\end{aligned}
$$

Then there is a non-zero $f \in A_{0}$ such that

$$
A \subseteq A_{0}\left[y, f^{-1}\right], \alpha_{1}, \ldots, \alpha_{n} \in A_{0}\left[y, f^{-1}\right]^{*}
$$$$
\operatorname{deg} f \leq(n+1)\left(2 d^{* *}\right)^{\exp O(r)}, h(f) \leq(n+1)\left(2 d^{* *}\right)^{\exp O(r)}\left(h^{* *}+1\right) .
$$

Proof. Take

$$
f:=\prod_{i=1}^{t} Q_{y_{i}} \cdot \prod_{j=1}^{n}\left(Q_{\alpha_{i}} Q_{\alpha_{i}^{-1}}\right) .
$$

Since in general, $Q_{\beta} \beta \in A_{0}[y]$ for $\beta \in K^{*}$, we have $f \beta \in A_{0}[y]$ for $\beta=$ $y_{1}, \ldots, y_{t}, \alpha_{1}, \alpha_{1}^{-1}, \ldots, \alpha_{n}, \alpha_{n}^{-1}$. This implies (3.14). The inequalities (3.15) follow at once from Lemmas 3.5 and 3.1 .

Lemma 3.7. Let $\lambda \in K^{*}$ and let $\varepsilon$ be a non-zero element of $A$. Let $(a, b)$ with $a, b \in \mathbb{Z}\left[X_{1}, \ldots, X_{r}\right]$ be a pair of representatives for $\lambda$. Put

$$
\begin{aligned}
& d_{0}:=\max \left(\operatorname{deg} f_{1}, \ldots, \operatorname{deg} f_{m}, \operatorname{deg} a, \operatorname{deg} b, \overline{\operatorname{deg}} \lambda \varepsilon\right), \\
& h_{0}:=\max \left(h\left(f_{1}\right), \ldots, h\left(f_{m}\right), h(a), h(b), \bar{h}(\lambda \varepsilon)\right) .
\end{aligned}
$$

Then $\varepsilon$ has a representative $\widetilde{\varepsilon} \in \mathbb{Z}\left[X_{1}, \ldots, X_{r}\right]$ such that

$$
\operatorname{deg} \widetilde{\varepsilon} \leq\left(2 d_{0}\right)^{\exp O\left(r \log ^{*} r\right)}\left(h_{0}+1\right), \quad h(\widetilde{\varepsilon}) \leq\left(2 d_{0}\right)^{\exp O\left(r \log ^{*} r\right)}\left(h_{0}+1\right)^{r+1} .
$$

If moreover $\varepsilon \in A^{*}$, then $\varepsilon^{-1}$ has a representative $\widetilde{\varepsilon} \in \mathbb{Z}\left[X_{1}, \ldots, X_{r}\right]$ with

$$
\operatorname{deg} \widetilde{\varepsilon} \leq\left(2 d_{0}\right)^{\exp O\left(r \log ^{*} r\right)}\left(h_{0}+1\right), \quad h(\widetilde{\varepsilon}) \leq\left(2 d_{0}\right)^{\exp O\left(r \log ^{*} r\right)}\left(h_{0}+1\right)^{r+1} .
$$


Proof. In case that $q>0$, we identify $z_{i}$ with $X_{i}$ and view elements of $A_{0}$ as polynomials in $\mathbb{Z}\left[X_{1}, \ldots, X_{q}\right]$. Put $Y:=\mathcal{G}_{0} \cdot\left(\sum_{i=1}^{t} a_{i} X_{q+i}\right)$. We have

$$
\lambda \varepsilon=Q^{-1} \sum_{i=0}^{D-1} P_{i} y^{i}
$$

with $P_{0}, \ldots, P_{D-1}, Q \in A_{0}$ and $\operatorname{gcd}\left(P_{0}, \ldots, P_{D-1}, Q\right)=1$. According to (3.16), $\widetilde{\varepsilon} \in \mathbb{Z}\left[X_{1}, \ldots, X_{r}\right]$ is a representative for $\varepsilon$ if and only if there are $g_{1}, \ldots, g_{m} \in \mathbb{Z}\left[X_{1}, \ldots, X_{r}\right]$ such that

$$
\widetilde{\varepsilon} \cdot(Q \cdot a)+\sum_{i=1}^{m} g_{i} f_{i}=b \sum_{i=0}^{D-1} P_{i} Y^{i} .
$$

We may view (3.17) as an inhomogeneous linear equation in the unknowns $\widetilde{\varepsilon}, g_{1}, \ldots, g_{m}$. Notice that by Lemmas 3.2 3.5 the degrees and logarith-

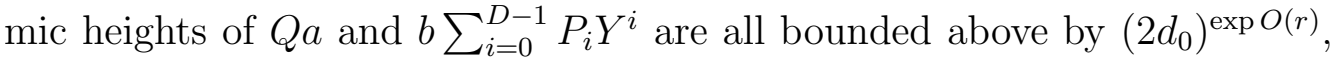
$\left(2 d_{0}\right)^{\exp O(r)}\left(h_{0}+1\right)$, respectively. Now Proposition 2.5 implies that (3.17) has a solution with upper bounds for $\operatorname{deg} \widetilde{\varepsilon}, h(\widetilde{\varepsilon})$ as stated in the lemma.

Now suppose that $\varepsilon \in A^{*}$. Again by (3.16), $\widetilde{\varepsilon} \in \mathbb{Z}\left[X_{1}, \ldots, X_{r}\right]$ is a representative for $\varepsilon^{-1}$ if and only if there are $g_{1}^{\prime}, \ldots, g_{m}^{\prime} \in \mathbb{Z}\left[X_{1}, \ldots, X_{r}\right]$ such that

$$
\widetilde{\varepsilon} \cdot b \sum_{i=0}^{D-1} P_{i} Y^{i}+\sum_{i=1}^{m} g_{i}^{\prime} f_{i}=Q \cdot a .
$$

Similarly as above, this equation has a solution with upper bounds for $\operatorname{deg} \widetilde{\varepsilon}$, $h(\widetilde{\varepsilon})$ as stated in the lemma.

Recall that we have defined $A_{0}=\mathbb{Z}\left[z_{1}, \ldots, z_{q}\right], K_{0}=\mathbb{Q}\left(z_{1}, \ldots, z_{q}\right)$ if $q>0$ and $A_{0}=\mathbb{Z}, K_{0}=\mathbb{Q}$ if $q=0$, and that in the case $q=0$, degrees and $\overline{\mathrm{deg}}$-s are always zero. Theorem 1.1 can be deduced from the following Proposition, which makes sense also if $q=0$. The proof of this Proposition is given in Sections 4 . 6 .

Proposition 3.8. Let $f \in A_{0}$ with $f \neq 0$, and let

$$
\mathcal{F}=X^{D}+\mathcal{F}_{1} X^{D-1}+\cdots+\mathcal{F}_{D} \in A_{0}[X] \quad(D \geq 1)
$$

be the minimal polynomial of $y$ over $K_{0}$. Let $d_{1} \geq 1, h_{1} \geq 1$ and suppose $\max \left(\operatorname{deg} f, \operatorname{deg} \mathcal{F}_{1}, \ldots, \operatorname{deg} \mathcal{F}_{D}\right) \leq d_{1}, \quad \max \left(h(f), h\left(\mathcal{F}_{1}\right), \ldots, h\left(\mathcal{F}_{D}\right)\right) \leq h_{1}$ 
Define the domain $B:=A_{0}\left[y, f^{-1}\right]$. Then for each pair $\left(\varepsilon_{1}, \eta_{1}\right)$ with

$$
\varepsilon_{1}+\eta_{1}=1, \quad \varepsilon_{1}, \eta_{1} \in B^{*}
$$

we have

$$
\begin{aligned}
& \overline{\operatorname{deg}} \varepsilon_{1}, \overline{\operatorname{deg}} \eta_{1} \leq 4 q D^{2} \cdot d_{1} \\
& \bar{h}\left(\varepsilon_{1}\right), \bar{h}\left(\eta_{1}\right) \leq \exp O\left(2 D\left(q+d_{1}\right) \log ^{*}\left\{2 D\left(q+d_{1}\right)\right\}+D h_{1}\right) .
\end{aligned}
$$

Proof of Theorem 1.1. Let $a, b, c \in A$ be the coefficients of (1.3), and $\widetilde{a}, \widetilde{b}, \widetilde{c}$ the representatives for $a, b, c$ from the statement of Theorem 1.1. By Lemma 3.6. there exists non-zero $f \in A_{0}$ such that that $A \subseteq B:=A_{0}\left[y, f^{-1}\right]$, $a, b, c \in B^{*}$, and moreover, $\operatorname{deg} f \leq(2 d)^{\exp O(r)}$ and $h(f) \leq(2 d)^{\exp O(r)}(h+1)$. By Corollary 3.4 we have the same type of upper bounds for the degrees and logarithmic heights of $\mathcal{F}_{1}, \ldots, \mathcal{F}_{D}$. So in Proposition 3.8 we may take $d_{1}=(2 d)^{\exp O(r)}, h_{1}=(2 d)^{\exp O(r)}(h+1)$. Finally, by Lemma 3.2 we have $D \leq d^{t}$.

Let $(\varepsilon, \eta)$ be a solution of (1.1) and put $\varepsilon_{1}:=a \varepsilon / c, \eta_{1}:=b \eta / c$. By Proposition 3.8 we have

$$
\overline{\operatorname{deg}} \varepsilon_{1} \leq 4 q d^{2 t}(2 d)^{\exp O(r)} \leq(2 d)^{\exp O(r)}, \quad \bar{h}\left(\varepsilon_{1}\right) \leq \exp \left((2 d)^{\exp O(r)}(h+1)\right) .
$$

We apply Lemma 3.7 with $\lambda=a / c$. Notice that $\lambda$ is represented by $(\widetilde{a}, \widetilde{c})$. By assumption, $\widetilde{a}$ and $\widetilde{c}$ have degrees at most $d$ and logarithmic heights at most $h$. Letting $\widetilde{a}, \widetilde{c}$ play the role of $a, b$ in Lemma 3.7, we see that in that lemma we may take $h_{0}=\exp \left((2 d)^{\exp O(r)}(h+1)\right)$ and $d_{0}=(2 d)^{\exp O(r)}$. It follows that $\varepsilon, \varepsilon^{-1}$ have representatives $\widetilde{\varepsilon}, \widetilde{\varepsilon} \in \mathbb{Z}\left[X_{1}, \ldots, X_{r}\right]$ such that

$$
\operatorname{deg} \widetilde{\varepsilon}, \operatorname{deg} \widetilde{\varepsilon}, h(\widetilde{\varepsilon}), h(\widetilde{\varepsilon}) \leq \exp \left((2 d)^{\exp O(r)}(h+1)\right) .
$$

We observe here that the upper bound for $\bar{h}\left(\varepsilon_{1}\right)$ dominates by far the other terms in our estimation. In the same manner one can derive similar upper bounds for the degrees and logarithmic heights of representatives for $\eta$ and $\eta^{-1}$. This completes the proof of Theorem 1.1 .

Proposition 3.8 is proved in Sections 46. In Section 4 we deduce the degree bound (3.19). Here, our main tool is Mason's effective result on $S$-unit equations over function fields [19, 1983]. In Section 5] we work out a more precise version of an effective specialization argument of Győry [8, 
1983], [9, 1984]. In Section [6 we prove (3.20) by combining the specialization argument from Section 5 with a recent effective result for $S$-unit equations over number fields, due to Györy an Yu [10, 2006].

\section{Bounding THE DEGREE}

We start with recalling some results on function fields in one variable. Let $\mathbf{k}$ be an algebraically closed field of characteristic 0 and let $z$ be transcendental over $\mathbf{k}$. Let $K$ be a finite extension of $\mathbf{k}(z)$. Denote by $g_{K / \mathbf{k}}$ the genus of $K$, and by $M_{K}$ the collection of valuations of $K / \mathbf{k}$, i.e, the valuations of $K$ with value group $\mathbb{Z}$ which are trivial on $\mathbf{k}$. Recall that these valuations satisfy the sum formula

$$
\sum_{v \in M_{K}} v(x)=0 \text { for } x \in K^{*} .
$$

As usual, for a finite subset $S$ of $M_{K}$ the group of $S$-units of $K$ is given by

$$
O_{S}^{*}=\left\{x \in K^{*}: v(x)=0 \text { for } v \in M_{K} \backslash S\right\} .
$$

The (homogeneous) height of $\mathbf{x}=\left(x_{1}, \ldots, x_{n}\right) \in K^{n}$ relative to $K / \mathbf{k}$ is defined by

$$
H_{K}(\mathbf{x})=H_{K}\left(x_{1}, \ldots, x_{n}\right):=-\sum_{v \in M_{K}} \min \left(v\left(x_{1}\right), \ldots, v\left(x_{n}\right)\right) .
$$

By the sum formula,

$$
H_{K}(\alpha \mathbf{x})=H_{K}(\mathbf{x}) \text { for } \alpha \in K^{*} .
$$

The height of $x \in K$ relative to $K / \mathbf{k}$ is defined by

$$
H_{K}(x):=H_{K}(1, x)=-\sum_{v \in M_{K}} \min (0, v(x)) .
$$

If $L$ is a finite extension of $K$, we have

$$
H_{L}\left(x_{1}, \ldots, x_{n}\right)=[L: K] H_{K}\left(x_{1}, \ldots, x_{n}\right) \text { for }\left(x_{1}, \ldots, x_{n}\right) \in K^{n} .
$$

By $\operatorname{deg} f$ we denote the total degree of $f \in \mathbf{k}[z]$. Then for $f_{1}, \ldots, f_{n} \in \mathbf{k}[z]$ with $\operatorname{gcd}\left(f_{1}, \ldots, f_{n}\right)=1$ we have

$$
H_{\mathbf{k}(z)}\left(f_{1}, \ldots, f_{n}\right)=\max \left(\operatorname{deg} f_{1}, \ldots, \operatorname{deg} f_{n}\right) .
$$


Lemma 4.1. Let $y_{1}, \ldots, y_{m} \in K$ and suppose that

$$
X^{m}+f_{1} X^{m-1}+\cdots+f_{m}=\left(X-y_{1}\right) \cdots\left(X-y_{m}\right)
$$

for certain $f_{1}, \ldots, f_{m} \in \mathbf{k}[z]$. Then

$$
[K: \mathbf{k}(z)] \max \left(\operatorname{deg} f_{1}, \ldots, \operatorname{deg} f_{m}\right)=\sum_{i=1}^{m} H_{K}\left(y_{i}\right) .
$$

Proof. By Gauss' Lemma we have for $v \in M_{K}$,

$$
\min \left(v\left(f_{1}\right), \ldots, v\left(f_{m}\right)\right)=\sum_{i=1}^{m} \min \left(0, v\left(y_{i}\right)\right) .
$$

Now take the sum over $v \in M_{K}$ and apply (4.2), (4.3).

Lemma 4.2. Let $K$ be the splitting field over $\mathbf{k}(z)$ of $F:=X^{m}+f_{1} X^{m-1}+$ $\cdots+f_{m}$, where $f_{1}, \ldots, f_{m} \in \mathbf{k}[z]$. Then

$$
g_{K / \mathbf{k}} \leq(d-1) m \cdot \max _{1 \leq i \leq m} \operatorname{deg} f_{i},
$$

where $d:=[K: \mathbf{k}(z)]$.

Proof. This is Lemma H of Schmidt [23, 1978].

In what follows, the cardinality of a set $S$ is denoted by $|S|$.

Proposition 4.3. Let $K$ be a finite extension of $\mathbf{k}(z)$ and $S$ be a finite subset of $M_{K}$. Then for every solution of

$$
x+y=1 \text { in } x, y \in O_{S}^{*} \backslash \mathbf{k}^{*}
$$

we have $\max \left(H_{K}(x), H_{K}(y)\right) \leq|S|+2 g_{K / \mathbf{k}}-2$.

Proof. See Mason [19, 1983].

We keep the notation from Proposition 3.8. We may assume that $q>0$ because the case $q=0$ is trivial. Let as before $K_{0}=\mathbb{Q}\left(z_{1}, \ldots, z_{q}\right), K=$ $K_{0}(y), A_{0}=\mathbb{Z}\left[z_{1}, \ldots, z_{q}\right], B=\mathbb{Z}\left[z_{1}, \ldots, z_{q}, f^{-1}, y\right]$.

Fix $i \in\{1, \ldots, q\}$. Let $\mathbf{k}_{i}:=\mathbb{Q}\left(z_{1}, \ldots, z_{i-1}, z_{i+1}, \ldots, z_{q}\right)$ and $\overline{\mathbf{k}_{i}}$ its algebraic closure. Thus, the domain $A_{0}$ is contained in $\overline{\mathbf{k}_{i}}\left[z_{i}\right]$. Let $y^{(1)}=$ $y, \ldots, y^{(D)}$ denote the conjugates of $y$ over $K_{0}$. Let $M_{i}$ denote the splitting field of the polynomial $X^{D}+\mathcal{F}_{1} X^{D-1}+\cdots+\mathcal{F}_{D}$ over $\overline{\mathbf{k}}_{i}\left(z_{i}\right)$, i.e.

$$
M_{i}:=\overline{\mathbf{k}_{i}}\left(z_{i}, y^{(1)}, \ldots, y^{(D)}\right) .
$$


The subring

$B_{i}:=\overline{\mathbf{k}_{i}}\left[z_{i}, f^{-1}, y^{(1)}, \ldots, y^{(D)}\right]$
of $M_{i}$ contains $B=\mathbb{Z}\left[z_{1}, \ldots, z_{q}, f^{-1}, y\right]$ as a subring. Put $\Delta_{i}:=\left[M_{i}: \overline{\mathbf{k}_{i}}\left(z_{i}\right)\right]$.

We apply Lemmas 4.1, 4.2 and Proposition 4.3 with $z_{i}, \mathbf{k}_{i}, M_{i}$ instead of $z, \mathbf{k}, K$. Denote by $g_{M_{i}}$ the genus of $M_{i} / \overline{\mathbf{k}_{i}}$. The height $H_{M_{i}}$ is taken with respect to $M_{i} / \overline{\mathbf{k}_{i}}$. For $g \in A_{0}$, we denote by $\operatorname{deg}_{z_{i}} g$ the degree of $g$ in the variable $z_{i}$.

Lemma 4.4. Let $\alpha \in K$ and denote by $\alpha^{(1)}, \ldots, \alpha^{(D)}$ the conjugates of $\alpha$ over $K_{0}$. Then

$$
\overline{\operatorname{deg}} \alpha \leq q D \cdot d_{1}+\sum_{i=1}^{q} \Delta_{i}^{-1} \sum_{j=1}^{D} H_{M_{i}}\left(\alpha^{(j)}\right) .
$$

Proof. We have

$$
\alpha=Q^{-1} \sum_{j=0}^{D-1} P_{j} y^{j}
$$

for certain $P_{0}, \ldots, P_{D-1}, Q \in A_{0}$ with $\operatorname{gcd}\left(Q, P_{0}, \ldots, P_{D-1}\right)=1$. Clearly,

$$
\overline{\operatorname{deg}} \alpha \leq \sum_{i=1}^{q} \mu_{i}, \quad \text { where } \mu_{i}:=\max \left(\operatorname{deg}_{z_{i}} Q, \operatorname{deg}_{z_{i}} P_{0}, \ldots, \operatorname{deg}_{z_{i}} P_{D-1}\right) .
$$

Below, we estimate $\mu_{1}, \ldots, \mu_{q}$ from above. We fix $i \in\{1, \ldots, q\}$ and use the notation introduced above.

Obviously,

$$
\alpha^{(k)}=Q^{-1} \sum_{j=0}^{D-1} P_{j} \cdot\left(y^{(k)}\right)^{j} \text { for } k=1, \ldots, D .
$$

Let $\Omega$ be the $D \times D$-matrix with rows

$$
(1, \ldots, 1),\left(y^{(1)}, \ldots, y^{(D)}\right), \ldots,\left(\left(y^{(1)}\right)^{D-1}, \ldots,\left(y^{(D)}\right)^{D-1}\right) .
$$

By Cramer's rule, $P_{j} / Q=\delta_{j} / \delta$, where $\delta=\operatorname{det} \Omega$, and $\delta_{j}$ is the determinant of the matrix obtained by replacing the $j$-th row of $\Omega$ by $\left(\alpha^{(1)}, \ldots, \alpha^{(D)}\right)$.

Gauss' Lemma implies that $\operatorname{gcd}\left(P_{0}, \ldots, P_{D-1}, Q\right)=1$ in the ring in $\mathbf{k}_{i}\left[z_{i}\right]$. By (4.3) (with $z_{i}$ in place of $z$ ) we have

$$
\begin{aligned}
\mu_{i} & =\max \left(\operatorname{deg}_{z_{i}} Q, \operatorname{deg}_{z_{i}} P_{0}, \ldots, \operatorname{deg}_{z_{i}} P_{D-1}\right) \\
& =H_{\overline{\mathbf{k}}\left(z_{i}\right)}\left(Q, P_{0}, \ldots, P_{D-1}\right) .
\end{aligned}
$$


Using $\left[M_{i}: \overline{\mathbf{k}_{i}}\left(z_{i}\right)\right]=\Delta_{i}$, the identities (4.2), (4.1) (with $z_{i}$ instead of $z$ ) and the fact that $\left(\delta, \delta_{1}, \ldots, \delta_{D}\right)$ is a scalar multiple of $\left(Q, P_{0}, \ldots, P_{D-1}\right)$ we obtain

$$
\Delta_{i} \mu_{i}=H_{M_{i}}\left(Q, P_{0}, \ldots, P_{D-1}\right)=H_{M_{i}}\left(\delta, \delta_{1}, \ldots, \delta_{D}\right) .
$$

We bound from above the right-hand side. A straightforward estimate yields that for every valuation $v$ of $M_{i} / \overline{\mathbf{k}_{i}}$,

$$
\begin{aligned}
-\min & \left(v(\delta), v\left(\delta_{1}\right), \ldots, v\left(\delta_{D}\right)\right) \\
& \leq-D \sum_{j=1}^{D} \min \left(0, v\left(y^{(j)}\right)\right)-\sum_{j=1}^{D} \min \left(0, v\left(\alpha^{(j)}\right)\right) .
\end{aligned}
$$

Then summation over $v$ and an application of Lemma 4.1 lead to

$$
\begin{aligned}
H_{M_{i}}\left(\delta, \delta_{1}, \ldots, \delta_{D}\right) & \leq D \sum_{j=1}^{D} H_{M_{i}}\left(y^{(j)}\right)+\sum_{j=1}^{D} H_{M_{i}}\left(\alpha^{(j)}\right) \\
& \leq D \Delta_{i} \max \left(\operatorname{deg}_{z_{i}} \mathcal{F}_{1}, \ldots, \operatorname{deg} \mathcal{F}_{D}\right)+\sum_{j=1}^{D} H_{M_{i}}\left(\alpha^{(j)}\right) \\
& \leq \Delta_{i} \cdot D d_{1}+\sum_{j=1}^{D} H_{M_{i}}\left(\alpha^{(j)}\right),
\end{aligned}
$$

and then a combination with (4.6) gives

$$
\mu_{i} \leq D d_{1}+\Delta_{i}^{-1} \sum_{j=1}^{D} H_{M_{i}}\left(\alpha^{(j)}\right) .
$$

Now these bounds for $i=1, \ldots, q$ together with (4.5) imply our Lemma.

Proof of (3.19). We fix again $i \in\{1, \ldots, q\}$ and use the notation introduced above. By Lemma 4.2, applied with $\mathbf{k}_{i}, z_{i}, M_{i}$ instead of $\mathbf{k}, z, K$ and with $F=\mathcal{F}=X^{D}+\mathcal{F}_{1} X^{D-1}+\cdots+\mathcal{F}_{D}$, we have

$$
g_{M_{i}} \leq\left(\Delta_{i}-1\right) D \max _{j} \operatorname{deg}_{z_{i}}\left(\mathcal{F}_{j}\right) \leq\left(\Delta_{i}-1\right) \cdot D d_{1} .
$$

Let $S$ denote the subset of valuations $v$ of $M_{i} / \overline{\mathbf{k}_{i}}$ such that $v\left(z_{i}\right)<0$ or $v(f)>0$. Each valuation of $\overline{\mathbf{k}_{i}}\left(z_{i}\right)$ can be extended to at most $\left[M_{i}: \overline{\mathbf{k}_{i}}\left(z_{i}\right)\right]=$ 
$\Delta_{i}$ valuations of $M_{i}$. Hence $M_{i}$ has at most $\Delta_{i}$ valuations $v$ with $v\left(z_{i}\right)<0$ and at most $\Delta_{i} \operatorname{deg} f$ valuations with $v(f)>0$. Thus,

$$
|S| \leq \Delta_{i}+\Delta_{i} \operatorname{deg}_{z_{i}} f \leq \Delta_{i}(1+\operatorname{deg} f) \leq \Delta_{i}\left(1+d_{1}\right)
$$

Every $\alpha \in M_{i}$ which is integral over $\overline{\mathbf{k}}_{i}\left[z_{i}, f^{-1}\right]$ belongs to $O_{S}$. The elements $y^{(1)}, \ldots, y^{(D)}$ belong to $M_{i}$ and are integral over $A_{0}=\mathbb{Z}\left[z_{1}, \ldots, z_{q}\right]$ so they certainly belong to $O_{S}$. As a consequence, the elements of $B$ and their conjugates over $\mathbb{Q}\left(z_{1}, \ldots, z_{q}\right)$ belong to $O_{S}$. In particular, if $\varepsilon_{1}, \eta_{1} \in B^{*}$ and $\varepsilon_{1}+\eta_{1}=1$, then

$$
\varepsilon_{1}^{(j)}+\eta_{1}^{(j)}=1, \varepsilon_{1}^{(j)}, \eta_{1}^{(j)} \in O_{S}^{*} \text { for } j=1, \ldots, D \text {. }
$$

We apply Proposition 4.3 and insert the upper bounds (4.7), (4.8). It follows that for $j=1, \ldots, D$ we have either $\varepsilon_{1}^{(j)} \in \overline{\mathbf{k}_{i}}$ or

$$
H_{M_{i}}\left(\varepsilon_{1}^{(j)}\right) \leq|S|+2 g_{M_{i}}-2 \leq 3 \Delta_{i} \cdot D d_{1} ;
$$

in fact the last upper bound is valid also if $\varepsilon_{1}^{(j)} \in \overline{\mathbf{k}_{i}}$. Together with Lemma 4.4 this gives

$$
\overline{\operatorname{deg}} \varepsilon_{1} \leq q D d_{1}+q D \cdot 3 D d_{1} \leq 4 q D^{2} d_{1} .
$$

For $\overline{\operatorname{deg}} \eta_{1}$ we derive the same estimate. This proves (3.19).

\section{Specializations}

In this section we prove some results about specialization homomorphisms from the domain $B$ from Proposition 3.8 to $\overline{\mathbb{Q}}$. We start with some notation and some preparatory lemmas.

The set of places of $\mathbb{Q}$ is $M_{\mathbb{Q}}=\{\infty\} \cup\{$ primes $\}$. By $|\cdot|_{\infty}$ we denote the ordinary absolute value on $\mathbb{Q}$ and by $|\cdot|_{p}$ ( $p$ prime) the $p$-adic absolute value, with $|p|_{p}=p^{-1}$. More generally, let $L$ be an algebraic number field and denote by $M_{L}$ its set of places. Given $v \in M_{L}$, we define the absolute value $|\cdot|_{v}$ in such a way that its restriction to $\mathbb{Q}$ is $|\cdot|_{p}$ if $v$ lies above $p \in M_{\mathbb{Q}}$. These absolute values satisfy the product formula $\prod_{v \in M_{L}}|x|_{v}^{d_{v}}=1$ for $x \in L^{*}$, where $d_{v}:=\left[L_{v}: \mathbb{Q}_{p}\right] /[L: \mathbb{Q}]$.

The (absolute logarithmic) height of $\mathbf{x}=\left(x_{1}, \ldots, x_{m}\right) \in L^{m} \backslash\{\mathbf{0}\}$ is defined by

$$
h(\mathbf{x})=h\left(x_{1}, \ldots, x_{m}\right)=\log \prod_{v \in M_{L}}\left(\max \left(\left|x_{1}\right|_{v}, \ldots,\left|x_{m}\right|_{v}\right)\right)^{d_{v}} .
$$


By the product formula, $h(\alpha \mathbf{x})=h(\mathbf{x})$ for $\alpha \in L^{*}$. Moreover, $h(\mathbf{x})$ depends only on $\mathbf{x}$ and not on the choice of the field $L$ such that $\mathbf{x} \in L^{m}$. So it defines a height on $\overline{\mathbb{Q}}^{m} \backslash\{\mathbf{0}\}$. The (absolute logarithmic) height of $\alpha \in \overline{\mathbb{Q}}$ is defined by $h(\alpha):=h((1, \alpha))$. In case that $\alpha \in L$ we have

$$
h(\alpha)=\log \prod_{v \in M_{L}} \max \left(1,|\alpha|_{v}^{d_{v}}\right) .
$$

For $\mathbf{a}=\left(a_{1}, \ldots, a_{m}\right) \in \mathbb{Z}^{m}$ with $\operatorname{gcd}\left(a_{1}, \ldots, a_{m}\right)=1$ we have

$$
h(\mathbf{a})=\log \max \left(\left|a_{1}\right|, \ldots,\left|a_{m}\right|\right) .
$$

It is easy to verify that for $a_{1}, \ldots, a_{m}, b_{1}, \ldots, b_{m} \in \overline{\mathbb{Q}}$,

$$
h\left(a_{1} b_{1}+\cdots+a_{m} b_{m}\right) \leq h\left(1, a_{1}, \ldots, a_{m}\right)+h\left(1, b_{1}, \ldots, b_{m}\right)+\log m .
$$

Let $G$ be a polynomial with coefficients in $L$. If $a_{1}, \ldots, a_{r}$ are the nonzero coefficients of $G$, we put $|G|_{v}:=\max \left(\left|a_{1}\right|_{v}, \ldots,\left|a_{r}\right|_{v}\right)$ for $v \in M_{L}$. For a polynomial $G$ with coefficients in $\mathbb{Z}$ we define $h(G):=\log |G|_{\infty}$.

We start with four auxiliary results that are used in the construction of our specializations.

Lemma 5.1. Let $m \geq 1, \alpha_{1}, \ldots, \alpha_{m} \in \overline{\mathbb{Q}}$ and suppose that $G(X):=$ $\prod_{i=1}^{m}\left(X-\alpha_{i}\right) \in \mathbb{Z}[X]$. Then

$$
\left|h(G)-\sum_{i=1}^{m} h\left(\alpha_{i}\right)\right| \leq m .
$$

Proof. See Bombieri and Gubler [3, Theorem 1.6.13, pp. 28].

Lemma 5.2. Let $m \geq 1$, let $\alpha_{1}, \ldots, \alpha_{m} \in \overline{\mathbb{Q}}$ be distinct and suppose that $G(X):=\prod_{i=1}^{m}\left(X-\alpha_{i}\right) \in \mathbb{Z}[X]$. Let $q, p_{0}, \ldots, p_{m-1}$ be integers with

$$
\operatorname{gcd}\left(q, p_{0}, \ldots, p_{m-1}\right)=1
$$

and put

$$
\beta_{i}:=\sum_{j=0}^{m-1}\left(p_{j} / q\right) \alpha_{i}^{j} \quad(i=1, \ldots, m)
$$

Then

$$
\log \max \left(|q|,\left|p_{0}\right|, \ldots,\left|p_{m-1}\right|\right) \leq 2 m^{2}+(m-1) h(G)+\sum_{j=1}^{m} h\left(\beta_{j}\right) .
$$


Proof. For $m=1$ the assertion is obvious, so we assume $m \geq 2$. Let $\Omega$ be the $m \times m$ matrix with rows $\left(\alpha_{1}^{i}, \ldots, \alpha_{m}^{i}\right)(i=0, \ldots, m-1)$. By Cramer's rule we have $p_{i} / q=\delta_{i} / \delta(i=0, \ldots, m-1)$, where $\delta=\operatorname{det} \Omega$ and $\delta_{i}$ is the determinant of the matrix, obtained by replacing the $i$-th row of $\Omega$ by $\left(\beta_{1}, \ldots, \beta_{m}\right)$. Put $\mu:=\log \max \left(|q|,\left|p_{0}\right|, \ldots,\left|p_{m-1}\right|\right)$. Then by (5.1),

$$
\mu=h\left(q, p_{0}, \ldots, p_{m-1}\right)=h\left(\delta, \delta_{0}, \ldots, \delta_{m-1}\right) .
$$

Let $L=\mathbb{Q}\left(\alpha_{1}, \ldots, \alpha_{m}\right)$. By Hadamard's inequality for the infinite places and the ultrametric inequality for the finite places, we get

$$
\max \left(|\delta|_{v},\left|\delta_{1}\right|_{v}, \ldots,\left|\delta_{m}\right|_{v}\right) \leq c_{v} \prod_{i=1}^{m} \max \left(1,\left|\alpha_{i}\right|_{v}\right)^{m-1} \max \left(1,\left|\beta_{i}\right|_{v}\right)
$$

for $v \in M_{L}$, where $c_{v}=m^{m / 2}$ if $v$ is infinite and $c_{v}=1$ if $v$ is finite. By taking the product over $v \in M_{L}$ and then logarithms, it follows that

$$
\mu \leq \frac{1}{2} m \log m+\sum_{i=1}^{m}\left((m-1) h\left(\alpha_{i}\right)+h\left(\beta_{i}\right)\right) .
$$

A combination with Lemma 5.1 implies our lemma.

Lemma 5.3. Let $g \in \mathbb{Z}\left[z_{1}, \ldots, z_{q}\right]$ be a non-zero polynomial of degree $d$ and $\mathcal{N}$ a subset of $\mathbb{Z}$ of cardinality $>d$. Then

$$
\left|\left\{\mathbf{u} \in \mathcal{N}^{q}: g(\mathbf{u})=0\right\}\right| \leq d|\mathcal{N}|^{q-1} .
$$

Proof. We proceed by induction on $q$. For $q=1$ the assertion is clear. Let $q \geq 2$. Write $g=\sum_{i=0}^{d_{0}} g_{i}\left(z_{1}, \ldots, z_{q-1}\right) z_{q}^{i}$ with $g_{i} \in \mathbb{Z}\left[z_{1}, \ldots, z_{q-1}\right]$ and $g_{d_{0}} \neq 0$. Then $\operatorname{deg} g_{d_{0}} \leq d-d_{0}$. By the induction hypothesis, there are at most $\left(d-d_{0}\right)|\mathcal{N}|^{q-2} \cdot|\mathcal{N}|$ tuples $\left(u_{1}, \ldots, u_{q}\right) \in \mathcal{N}^{q}$ with $g_{d_{0}}\left(u_{1}, \ldots, u_{q-1}\right)=0$. Further, there are at most $|\mathcal{N}|^{q-1} \cdot d_{0}$ tuples $\mathbf{u} \in \mathcal{N}^{q}$ with $g_{d_{0}}\left(u_{1}, \ldots, u_{q-1}\right) \neq$ 0 and $g\left(u_{1}, \ldots, u_{q}\right)=0$. Summing these two quantities implies that $g$ has at most $d|\mathcal{N}|^{q-1}$ zeros in $\mathcal{N}^{q}$.

Lemma 5.4. Let $g_{1}, g_{2} \in \mathbb{Z}\left[z_{1}, \ldots, z_{q}\right]$ be two non-zero polynomials of degrees $D_{1}, D_{2}$, respectively, and let $N$ be an integer $\geq \max \left(D_{1}, D_{2}\right)$. Define

$$
\mathcal{S}:=\left\{\mathbf{u} \in \mathbb{Z}^{q}:|\mathbf{u}| \leq N, g_{2}(\mathbf{u}) \neq 0\right\} .
$$

Then $\mathcal{S}$ is non-empty, and

$$
\begin{aligned}
& \left|g_{1}\right|_{p} \leq(4 N)^{q D_{1}\left(D_{1}+1\right) / 2} \max \left\{\left|g_{1}(\mathbf{u})\right|_{p}: \mathbf{u} \in \mathcal{S}\right\} \\
& \qquad \text { for } p \in M_{\mathbb{Q}}=\{\infty\} \cup\{\text { primes }\} .
\end{aligned}
$$


Proof. Put $C_{p}:=\max \left\{\left|g_{1}(\mathbf{u})\right|_{p}: \quad \mathbf{u} \in \mathcal{S}\right\}$ for $p \in M_{\mathbb{Q}}$. We proceed by induction on $q$, starting with $q=0$. In the case $q=0$ we interpret $g_{1}, g_{2}$ as non-zero constants with $\left|g_{1}\right|_{p}=C_{p}$ for $p \in M_{\mathbb{Q}}$. Then the lemma is trivial. Let $q \geq 1$. Write

$$
g_{1}=\sum_{j=0}^{D_{1}^{\prime}} g_{1 j}\left(z_{1}, \ldots, z_{q-1}\right) z_{q}^{j}, \quad g_{2}=\sum_{j=0}^{D_{2}^{\prime}} g_{2 j}\left(z_{1}, \ldots, z_{q-1}\right) z_{q}^{j},
$$

where $g_{1, D_{1}^{\prime}}, g_{2, D_{2}^{\prime}} \neq 0$. By the induction hypothesis, the set

$$
\mathcal{S}^{\prime}:=\left\{\mathbf{u}^{\prime} \in \mathbb{Z}^{q-1}:\left|\mathbf{u}^{\prime}\right| \leq N, g_{2, D_{2}^{\prime}}\left(\mathbf{u}^{\prime}\right) \neq 0\right\}
$$

is non-empty and moreover,

$$
\max _{0 \leq j \leq D_{1}^{\prime}}\left|g_{1 j}\right|_{p} \leq(4 N)^{(q-1) D_{1}\left(D_{1}+1\right) / 2} C_{p}^{\prime} \text { for } p \in M_{\mathbb{Q}}
$$

where

$$
C_{p}^{\prime}:=\max \left\{\left|g_{1 j}\left(\mathbf{u}^{\prime}\right)\right|_{p}: \mathbf{u}^{\prime} \in \mathcal{S}^{\prime}, j=0, \ldots, D_{1}^{\prime}\right\} .
$$

We estimate $C_{p}^{\prime}$ from above in terms of $C_{p}$. Fix $\mathbf{u}^{\prime} \in \mathcal{S}^{\prime}$. There are at least $2 N+1-D_{2}^{\prime} \geq D_{1}^{\prime}+1$ integers $u_{q}$ with $\left|u_{q}\right| \leq N$ such that $g_{2}\left(\mathbf{u}^{\prime}, u_{q}\right) \neq 0$. Let $a_{0}, \ldots, a_{D_{1}^{\prime}}$ be distinct integers from this set. By Lagrange's interpolation formula,

$$
\begin{aligned}
g_{1}\left(\mathbf{u}^{\prime}, X\right) & =\sum_{j=0}^{D_{1}^{\prime}} g_{1 j}\left(\mathbf{u}^{\prime}\right) X^{j} \\
& =\sum_{j=0}^{D_{1}^{\prime}} g_{1}\left(\mathbf{u}^{\prime}, a_{j}\right) \prod_{\substack{i=0 \\
i \neq j}}^{D_{1}^{\prime}} \frac{X-a_{i}}{a_{j}-a_{i}} .
\end{aligned}
$$

From this we deduce

$$
\begin{aligned}
\max _{0 \leq j \leq D_{1}^{\prime}}\left|g_{1 j}\left(\mathbf{u}^{\prime}\right)\right| & \leq C_{\infty} \sum_{j=0}^{D_{1}^{\prime}} \prod_{\substack{i=0 \\
i \neq j}}^{D_{1}^{\prime}} \frac{1+\left|a_{i}\right|}{\left|a_{j}-a_{i}\right|} \\
& \leq C_{\infty}\left(D_{1}^{\prime}+1\right)(N+1)^{D_{1}^{\prime}} \leq(4 N)^{D_{1}^{\prime}\left(D_{1}^{\prime}+1\right) / 2} C_{\infty} .
\end{aligned}
$$

Now let $p$ be a prime and put $\Delta:=\prod_{1 \leq i<j \leq D_{1}^{\prime}}\left|a_{j}-a_{i}\right|$. Then

$$
\max _{0 \leq j \leq D_{1}^{\prime}}\left|g_{1 j}\left(\mathbf{u}^{\prime}\right)\right|_{p} \leq C_{p}|\Delta|_{p}^{-1} \leq \Delta C_{p} \leq(4 N)^{D_{1}^{\prime}\left(D_{1}^{\prime}+1\right) / 2} C_{p}
$$


It follows that $C_{p}^{\prime} \leq(4 N)^{D_{1}^{\prime}\left(D_{1}^{\prime}+1\right) / 2} C_{p}$ for $p \in M_{\mathbb{Q}}$. A combination with (5.4) gives (5.3)

We now introduce our specializations $B \rightarrow \overline{\mathbb{Q}}$ and prove some properties. We assume $q>0$ and apart from that keep the notation and assumptions from Proposition 3.8. In particular, $A_{0}=\mathbb{Z}\left[z_{1}, \ldots, z_{q}\right], K_{0}=\mathbb{Q}\left(z_{1}, \ldots, z_{q}\right)$ and

$$
K=\mathbb{Q}\left(z_{1}, \ldots, z_{q}, y\right), \quad B=\mathbb{Z}\left[z_{1}, \ldots, z_{q}, f^{-1}, y\right],
$$

where $f$ is a non-zero element of $A_{0}, y$ is integral over $A_{0}$, and $y$ has minimal polynomial

$$
\mathcal{F}:=X^{D}+\mathcal{F}_{1} X^{D-1}+\cdots+\mathcal{F}_{D} \in A_{0}[X]
$$

over $K_{0}$. In the case $D=1$, we take $y=1, \mathcal{F}=X-1$.

To allow for other applications (e.g., Lemma 7.2 below), we consider a more general situation than what is needed for the proof of Proposition 3.8 . Let $d_{1} \geq d_{0} \geq 1, h_{1} \geq h_{0} \geq 1$ and assume that

$$
\left\{\begin{array}{l}
\max \left(\operatorname{deg} \mathcal{F}_{1}, \ldots, \operatorname{deg} \mathcal{F}_{D}\right) \leq d_{0}, \quad \max \left(d_{0}, \operatorname{deg} f\right) \leq d_{1}, \\
\max \left(h\left(\mathcal{F}_{1}\right), \ldots, h\left(\mathcal{F}_{D}\right)\right) \leq h_{0}, \quad \max \left(h_{0}, h(f)\right) \leq h_{1} .
\end{array}\right.
$$

Let $\mathbf{u}=\left(u_{1}, \ldots, u_{q}\right) \in \mathbb{Z}^{q}$. Then the substitution $z_{1} \mapsto u_{1}, \ldots, z_{q} \mapsto u_{q}$ defines a ring homomorphism (specialization)

$$
\varphi_{\mathbf{u}}: \alpha \mapsto \alpha(\mathbf{u}):\left\{g_{1} / g_{2}: g_{1}, g_{2} \in A_{0}, g_{2}(\mathbf{u}) \neq 0\right\} \rightarrow \mathbb{Q}
$$

We want to extend this to a ring homomorphism from $B$ to $\overline{\mathbb{Q}}$ and for this, we have to impose some restrictions on $\mathbf{u}$. Denote by $\Delta_{\mathcal{F}}$ the discriminant of $\mathcal{F}$ (with $\Delta_{\mathcal{F}}:=1$ if $D=\overline{\operatorname{deg}} \mathcal{F}=1$ ), and let

$$
\mathcal{H}:=\Delta_{\mathcal{F}} \mathcal{F}_{D} \cdot f \text {. }
$$

Then $\mathcal{H} \in A_{0}$. Using that $\Delta_{\mathcal{F}}$ is a polynomial of degree $2 D-2$ with integer coefficients in $\mathcal{F}_{1}, \ldots, \mathcal{F}_{D}$, it follows easily that

$$
\operatorname{deg} \mathcal{H} \leq(2 D-1) d_{0}+d_{1} \leq 2 D d_{1}
$$

Now assume that

$$
\mathcal{H}(\mathbf{u}) \neq 0 .
$$

Then $f(\mathbf{u}) \neq 0$ and moreover, the polynomial

$$
\mathcal{F}_{\mathbf{u}}:=X^{D}+\mathcal{F}_{1}(\mathbf{u}) X^{D-1}+\cdots+\mathcal{F}_{D}(\mathbf{u})
$$


has $D$ distinct zeros which are all different from 0 , say $y_{1}(\mathbf{u}), \ldots, y_{D}(\mathbf{u})$. Thus, for $j=1, \ldots, D$ the assignment

$$
z_{1} \mapsto u_{1}, \ldots, z_{q} \mapsto u_{q}, \quad y \mapsto y_{j}(\mathbf{u})
$$

defines a ring homomorphism $\varphi_{\mathbf{u}, j}$ from $B$ to $\overline{\mathbb{Q}}$; in the case $D=1$ it is just $\varphi_{\mathbf{u}}$. The image of $\alpha \in B$ under $\varphi_{\mathbf{u}, j}$ is denoted by $\alpha_{j}(\mathbf{u})$. Recall that we may express elements $\alpha$ of $B$ as

$$
\begin{aligned}
\alpha= & \sum_{i=0}^{D-1}\left(P_{i} / Q\right) y^{i} \\
& \text { with } P_{0}, \ldots, P_{D-1}, Q \in A_{0}, \operatorname{gcd}\left(P_{0}, \ldots, P_{D-1}, Q\right)=1 .
\end{aligned}
$$

Since $\alpha \in B$, the denominator $Q$ must divide a power of $f$, hence $Q(\mathbf{u}) \neq 0$. So we have

$$
\alpha_{j}(\mathbf{u})=\sum_{i=0}^{D-1}\left(P_{i}(\mathbf{u}) / Q(\mathbf{u})\right) y_{j}(\mathbf{u})^{i} \quad(j=1, \ldots, D) .
$$

It is obvious that $\varphi_{\mathbf{u}, j}$ is the identity on $B \cap \mathbb{Q}$. Thus, if $\alpha \in B \cap \overline{\mathbb{Q}}$, then $\varphi_{\mathbf{u}, j}(\alpha)$ has the same minimal polynomial as $\alpha$ and so it is conjugate to $\alpha$.

For $\mathbf{u}=\left(u_{1}, \ldots, u_{q}\right) \in \mathbb{Z}^{q}$, we put $|\mathbf{u}|:=\max \left(\left|u_{1}\right|, \ldots,\left|u_{q}\right|\right)$. It is easy to verify that for any $g \in A_{0}, \mathbf{u} \in \mathbb{Z}^{q}$,

$$
\log |g(\mathbf{u})| \leq q \log \operatorname{deg} g+h(g)+\operatorname{deg} g \log \max (1,|\mathbf{u}|) .
$$

In particular,

$$
h\left(\mathcal{F}_{\mathbf{u}}\right) \leq q \log d_{0}+h_{0}+d_{0} \log \max (1,|\mathbf{u}|)
$$

and so by Lemma 5.2 (ii),

$$
\sum_{j=1}^{D} h\left(y_{j}(\mathbf{u})\right) \leq D+q \log d_{0}+h_{0}+d_{0} \log \max (1,|\mathbf{u}|) .
$$

Define the algebraic number fields $K_{\mathbf{u}, j}:=\mathbb{Q}\left(\mathbf{y}_{j}(\mathbf{u})\right)(j=1, \ldots, D)$. Denote by $\Delta_{L}$ the discriminant of an algebraic number field $L$. We derive an upper bound for the discriminant $\Delta_{K_{\mathbf{u}, j}}$ of $K_{\mathbf{u}, j}$.

Lemma 5.5. Let $\mathbf{u} \in \mathbb{Z}^{q}$ with $\mathcal{H}(\mathbf{u}) \neq 0$. Then for $j=1, \ldots, D$ we have $\left[K_{\mathbf{u}, j}: \mathbb{Q}\right] \leq D$ and

$$
\left|\Delta_{K_{\mathbf{u}, j}}\right| \leq D^{2 D-1}\left(d_{0}^{q} \cdot e^{h_{0}} \max (1,|\mathbf{u}|)^{d_{0}}\right)^{2 D-2} .
$$


Proof. Let $j \in\{1, \ldots, D\}$. The estimate for the degree is obvious. To estimate the discriminant, let $\mathcal{P}_{j}$ be the monic minimal polynomial of $y_{j}(\mathbf{u})$. Then $\Delta_{K_{\mathbf{u}, j}}$ divides the discriminant $\Delta_{\mathcal{P}_{j}}$ of $\mathcal{P}_{j}$. Using the expression of the discriminant of a monic polynomial as the product of the squares of the differences of its zeros, one easily shows that $\Delta_{\mathcal{P}_{j}}$ divides $\Delta_{\mathcal{F}_{\mathbf{u}}}$ in the ring of algebraic integers and so also in $\mathbb{Z}$. Therefore, $\Delta_{K_{\mathbf{u}}, j}$ divides $\Delta_{\mathcal{F}_{\mathbf{u}}}$ in $\mathbb{Z}$.

It remains to estimate from above the discriminant of $\mathcal{F}_{\mathbf{u}}$. By, e.g., Lewis and Mahler [14, bottom of p. 335], we have

$$
\left|\Delta_{\mathcal{F}_{\mathbf{u}}}\right| \leq D^{2 D-1}\left|\mathcal{F}_{\mathbf{u}}\right|^{2 D-2}
$$

where $\left|\mathcal{F}_{\mathbf{u}}\right|$ denotes the maximum of the absolute values of the coefficients of $\mathcal{F}_{\mathbf{u}}$. By (15.12), this is bounded above by $d_{0}^{q} e^{h_{0}} \max (1,|\mathbf{u}|)^{d_{0}}$, so

$$
\left|\Delta_{\mathcal{F}_{\mathbf{u}}}\right| \leq D^{2 D-1}\left(d_{0}^{q} e^{h_{0}} \max (1,|\mathbf{u}|)^{d_{0}}\right)^{2 D-2} .
$$

This implies our lemma.

We finish with two lemmas, which relate the height of $\alpha \in B$ to the heights of $\alpha_{j}(\mathbf{u})$ for $\mathbf{u} \in \mathbb{Z}^{q}$.

Lemma 5.6. Let $\mathbf{u} \in \mathbb{Z}^{q}$ with $\mathcal{H}(\mathbf{u}) \neq 0$. Let $\alpha \in B$. Then for $j=1, \ldots, D$,

$$
\begin{aligned}
h\left(\alpha_{j}(\mathbf{u})\right) \leq D^{2}+q\left(D \log d_{0}+\log \overline{\operatorname{deg}} \alpha\right) & +D h_{0}+\bar{h}(\alpha)+ \\
& +\left(D d_{0}+\overline{\operatorname{deg}} \alpha\right) \log \max (1,|\mathbf{u}|)
\end{aligned}
$$

Proof. Let $P_{0}, \ldots, P_{D-1}, Q$ as in (5.9) and write $\alpha_{j}(\mathbf{u})$ as in (5.10). By (5.2),

$$
\begin{aligned}
& h\left(\alpha_{j}(\mathbf{u})\right) \leq \log D+ \\
& \quad+h\left(1, P_{0}(\mathbf{u}) / Q(\mathbf{u}), \ldots, P_{D-1}(\mathbf{u}) / Q(\mathbf{u})\right)+(D-1) h\left(y_{j}(\mathbf{u})\right) .
\end{aligned}
$$

From (5.11) we infer

$$
\begin{aligned}
h(1, & \left.P_{0}(\mathbf{u}) / Q(\mathbf{u}), \ldots, P_{D-1}(\mathbf{u}) / Q(\mathbf{u})\right) \\
& \leq \log \max \left(|Q(\mathbf{u})|,\left|P_{0}(\mathbf{u})\right|, \ldots,\left|P_{D-1}(\mathbf{u})\right|\right) \\
& \leq q \log \overline{\operatorname{deg}} \alpha+\bar{h}(\alpha)+\overline{\operatorname{deg}} \alpha \cdot \log \max (1,|\mathbf{u}|) .
\end{aligned}
$$

By combining (5.14) with this inequality and with (5.13), our lemma easily follows. 
Lemma 5.7. Let $\alpha \in B, \alpha \neq 0$, and let $N$ be an integer with

$$
N \geq \max \left(\overline{\operatorname{deg}} \alpha, 2 D d_{0}+2(q+1)\left(d_{1}+1\right)\right) .
$$

Then the set

$$
\mathcal{S}:=\left\{\mathbf{u} \in \mathbb{Z}^{q}:|\mathbf{u}| \leq N, \mathcal{H}(\mathbf{u}) \neq 0\right\}
$$

is non-empty, and

$$
\bar{h}(\alpha) \leq 5 N^{4}\left(h_{1}+1\right)^{2}+2 D\left(h_{1}+1\right) H
$$

where $H:=\max \left\{h\left(\alpha_{j}(\mathbf{u})\right): \mathbf{u} \in \mathcal{S}, j=1, \ldots, D\right\}$.

Proof. It follows from our assumption on $N$, (5.7), and Lemma 5.4 that $\mathcal{S}$ is non-empty. We proceed with estimating $\bar{h}(\alpha)$.

Let $P_{0}, \ldots, P_{D-1}, Q \in A_{0}$ be as in (5.9). We analyse $Q$ more closely. Let

$$
f= \pm p_{1}^{k_{1}} \cdots p_{m}^{k_{m}} g_{1}^{l_{1}} \cdots g_{n}^{l_{n}}
$$

be the unique factorization of $f$ in $A_{0}$, where $p_{1}, \ldots, p_{m}$ are distinct prime numbers, and $\pm g_{1}, \ldots, \pm g_{n}$ distinct irreducible elements of $A_{0}$ of positive degree. Notice that

$$
\begin{gathered}
m \leq h(f) / \log 2 \leq h_{1} / \log 2, \\
\sum_{i=1}^{n} l_{i} h\left(g_{i}\right) \leq q d_{1}+h_{1},
\end{gathered}
$$

where the last inequality is a consequence of Lemma 5.1. Since $\alpha \in B$, the polynomial $Q$ is also composed of $p_{1}, \ldots, p_{m}, g_{1}, \ldots, g_{n}$. Hence

$$
Q=a \widetilde{Q} \text { with } a= \pm p_{1}^{k_{1}^{\prime}} \cdots p_{m}^{k_{m}^{\prime}}, \widetilde{Q}=g_{1}^{l_{1}^{\prime}} \cdots g_{n}^{l_{n}^{\prime}}
$$

for certain non-negative integers $k_{1}^{\prime}, \ldots, l_{n}^{\prime}$. Clearly,

$$
l_{1}^{\prime}+\cdots+l_{n}^{\prime} \leq \operatorname{deg} Q \leq \overline{\operatorname{deg}} \alpha \leq N
$$

and by Lemma 3.1 and (5.16),

$$
h(\widetilde{Q}) \leq q \operatorname{deg} Q+\sum_{i=1}^{n} l_{i}^{\prime} h\left(g_{i}\right) \leq N\left(q+q d_{1}+h_{1}\right) \leq N^{2}\left(h_{1}+1\right) .
$$

In view of (5.11), we have for $\mathbf{u} \in \mathcal{S}$,

$$
\begin{aligned}
\log |\widetilde{Q}(\mathbf{u})| & \leq q \log d_{1}+h(\widetilde{Q})+\operatorname{deg} Q \log N \\
& \leq \frac{3}{2} N \log N+N^{2}\left(h_{1}+1\right) \leq N^{2}\left(h_{1}+2\right) .
\end{aligned}
$$


Hence

$$
h\left(\widetilde{Q}(\mathbf{u}) \alpha_{j}(\mathbf{u})\right) \leq N^{2}\left(h_{1}+2\right)+H
$$

for $\mathbf{u} \in \mathcal{S}, j=1, \ldots, D$. Further, by (5.10), (5.16) we have

$$
\widetilde{Q}(\mathbf{u}) \alpha_{j}(\mathbf{u})=\sum_{i=0}^{D-1}\left(P_{i}(\mathbf{u}) / a\right) y_{j}(\mathbf{u})^{i} .
$$

Put

$$
\delta(\mathbf{u}):=\operatorname{gcd}\left(a, P_{0}(\mathbf{u}), \ldots, P_{D-1}(\mathbf{u})\right) .
$$

Then by applying Lemma 5.2 and then $(5.12)$ we obtain

$$
\begin{aligned}
& \log \left(\frac{\max \left(|a|,\left|P_{0}(\mathbf{u})\right|, \ldots,\left|P_{D-1}(\mathbf{u})\right|\right.}{\delta(\mathbf{u})}\right) \\
& \leq 2 D^{2}+(D-1) h\left(\mathcal{F}_{\mathbf{u}}\right)+D\left(N^{2}\left(h_{1}+2\right)+H\right) \\
& \leq 2 D^{2}+(D-1)\left(q \log d_{1}+h_{1}+d_{1} \log N\right)+D\left(N^{2}\left(h_{1}+2\right)+H\right) \\
& \leq N^{3}\left(h_{1}+2\right)+D H
\end{aligned}
$$

Our assumption that $\operatorname{gcd}\left(Q, P_{0}, \ldots, P_{D-1}\right)=1$ implies that the gcd of $a$ and the coefficients of $P_{0}, \ldots, P_{D-1}$ is 1 . Let $p \in\left\{p_{1}, \ldots, p_{m}\right\}$ be one of the prime factors of $a$. There is $j \in\{0, \ldots, D-1\}$ such that $\left|P_{j}\right|_{p}=1$. Our assumption on $N$ and (5.7) imply that $N \geq \max \left(\operatorname{deg} \mathcal{H}, \operatorname{deg} P_{j}\right)$. This means that Lemma 5.4 is applicable with $g_{1}=P_{j}$ and $g_{2}=\mathcal{H}$. It follows that

$$
\max \left\{\left|P_{j}(\mathbf{u})\right|_{p}: \quad \mathbf{u} \in \mathcal{S}\right\} \geq(4 N)^{-q N(N+1) / 2} .
$$

That is, there is $\mathbf{u}_{0} \in \mathcal{S}$ with $\left|P_{j}\left(\mathbf{u}_{0}\right)\right|_{p} \geq(4 N)^{-q N(N+1) / 2}$. Hence

$$
\left|\delta\left(\mathbf{u}_{0}\right)\right|_{p} \geq(4 N)^{-q N(N+1) / 2} .
$$

Together with (5.19), this implies

$$
\begin{aligned}
\log |a|_{p}^{-1} & \leq \log \left|a / \delta\left(\mathbf{u}_{0}\right)\right|+\log \left|\delta\left(\mathbf{u}_{0}\right)\right|_{p}^{-1} \\
& \leq N^{3}\left(h_{1}+2\right)+D H+\frac{1}{2} N^{3} \log 4 N \leq N^{4}\left(h_{1}+3\right)+D H
\end{aligned}
$$

Combining this with the upper bound $(5.15)$ for the number of prime factors of $a$, we obtain

$$
\log |a| \leq 2 N^{4} h_{1}\left(h_{1}+3\right)+2 D h_{1} \cdot H
$$


Together with (5.17), (5.18), this implies

$$
\begin{aligned}
h(Q) & \leq 2 N^{4} h_{1}\left(h_{1}+3\right)+2 D h_{1} \cdot H+N^{2}\left(h_{1}+1\right) \\
& \leq 3 N^{4}\left(h_{1}+1\right)^{2}+2 D h_{1} \cdot H .
\end{aligned}
$$

Further, the right-hand side of (5.20) is also an upper bound for $\log \delta(\mathbf{u})$, for $\mathbf{u} \in \mathcal{S}$. Combining this with (5.19) gives

$$
\begin{aligned}
& \log \max \left\{\left|P_{j}(\mathbf{u})\right|: \mathbf{u} \in \mathcal{S}, j=0, \ldots, D-1\right\} \\
& \quad \leq N^{3}\left(h_{1}+2\right)+D H+3 N^{4}\left(h_{1}+1\right)^{2}+2 D h_{1} \cdot H \\
& \quad \leq 4 N^{4}\left(h_{1}+1\right)^{2}+2 D\left(h_{1}+1\right) \cdot H .
\end{aligned}
$$

Another application of Lemma 5.4 yields

$$
\begin{aligned}
h\left(P_{j}\right) & \leq \frac{1}{2} q N(N+1) \log 4 N+4 N^{4}\left(h_{1}+1\right)^{2}+2 D\left(h_{1}+1\right) \cdot H \\
& \leq 5 N^{4}\left(h_{1}+1\right)^{2}+2 D\left(h_{1}+1\right) \cdot H
\end{aligned}
$$

for $j=0, \ldots, D-1$. Together with (5.21) this gives the upper bound for $\bar{h}(\alpha)$ from our lemma.

\section{Completion of the proof of Proposition 3.8}

It remains only to prove the height bound in (3.20). We use an effective result of Győry and $\mathrm{Yu}$ [10, 2006] on $S$-unit equations in number fields. To state this, we need some notation.

Let $L$ be an algebraic number field of degree $d_{L}$. We denote by $O_{L}, M_{L}$, $\Delta_{L}, h_{L}, R_{L}$ the ring of integers, set of places, discriminant, class number and regulator of $L$. The norm of an ideal $\mathfrak{a}$ of $O_{L}$, i.e., $\left|O_{L} / \mathfrak{a}\right|$, is denoted by $N \mathfrak{a}$.

Further, let $S$ be a finite set of places of $L$, containing all infinite places. Suppose $S$ has cardinality $s$. Recall that the ring of $S$-integers $O_{S}$ and the group of $S$-units $O_{S}^{*}$ are given by

$$
\begin{aligned}
& O_{S}=\left\{x \in L:|x|_{v} \leq 1 \text { for } v \in M_{L} \backslash S\right\}, \\
& O_{S}^{*}=\left\{x \in L:|x|_{v}=1 \text { for } v \in M_{L} \backslash S\right\} .
\end{aligned}
$$

If case that $S$ consists only of the infinite places of $L$, we put $P:=2$, $Q:=2$. If $S$ contains also finite places, let $\mathfrak{p}_{1}, \ldots, \mathfrak{p}_{t}$ denote the prime 
ideals corresponding to the finite places of $S$, and put

$$
P:=\max \left\{N \mathfrak{p}_{1}, \ldots, N \mathfrak{p}_{t}\right\}, \quad Q:=N\left(\mathfrak{p}_{1} \cdots \mathfrak{p}_{t}\right) .
$$

Further, let $R_{S}$ denote the $S$-regulator associated with $S$. In case that $S$ consists only of the infinite places of $L$ it is equal to $R_{L}$, while otherwise

$$
R_{S}=h_{S} R_{L} \prod_{i=1}^{t} \log N \mathfrak{p}_{i}
$$

where $h_{S}$ is a divisor of $h_{L}$ whose definition is not important here. By, e.g., formula (59) of [10] (which is an easy consequence of formula (2) of Louboutin [16, 2000]) we have

$$
h_{L} R_{L} \leq\left|\Delta_{L}\right|^{1 / 2}\left(\log ^{*}\left|\Delta_{L}\right|\right)^{d_{L}-1} .
$$

By the inequality of the geometric and arithmetic mean, we have for $t>0$,

$$
\prod_{i=1}^{t} \log N \mathfrak{p}_{i} \leq\left(t^{-1} \log \left(N \mathfrak{p}_{1} \cdots N \mathfrak{p}_{t}\right)\right)^{t} \leq(\log Q)^{s}
$$

and hence,

$$
R_{S} \leq\left|\Delta_{L}\right|^{1 / 2}\left(\log ^{*}\left|\Delta_{L}\right|\right)^{d_{L}-1} \cdot\left(\log ^{*} Q\right)^{s} .
$$

This is clearly true also if $t=0$.

Proposition 6.1. Let $\varepsilon, \eta$ such that

$$
\varepsilon+\eta=1, \quad \varepsilon, \eta \in O_{S}^{*} .
$$

Then

$$
\max (h(\varepsilon), h(\eta)) \leq c_{1} P R_{S}\left(1+\log ^{*} R_{S} / \log P\right)
$$

where

$$
c_{1}=\max \left(1, \pi / d_{L}\right) s^{2 s+3.5} 2^{7 s+27}(\log 2 s) d_{L}^{2(s+1)}\left(\log ^{*} 2 d_{L}\right)^{3} .
$$

Proof. This is Theorem 1 of Györy, Yu [10] with $\alpha=\beta=1$.

Proof of (3.20). As before, we use $O(\cdot)$ to denote a quantity which is $c \times$ the expression between the parentheses, where $c$ is an effectively computable absolute constant which may be different at each occurrence of the $O$-symbol.

We first consider the case $q>0$. Let $\varepsilon_{1}, \eta_{1}$ be a solution of (3.18). Pick $\mathbf{u} \in \mathbb{Z}^{q}$ with $\mathcal{H}(\mathbf{u}) \neq 0$, pick $j \in\{1, \ldots, D\}$ and put $L:=K_{\mathbf{u}, j}$. Further, let the set of places $S$ consist of all infinite places of $L$, and all finite places 
of $L$ lying above the rational prime divisors of $f(\mathbf{u})$. Note that $y_{j}(\mathbf{u})$ is an algebraic integer, and $f(\mathbf{u}) \in O_{S}^{*}$. Hence $\varphi_{\mathbf{u}, j}(B) \subseteq O_{S}$ and $\varphi_{\mathbf{u}, j}\left(B^{*}\right) \subseteq O_{S}^{*}$. So

$$
\varepsilon_{1, j}(\mathbf{u})+\eta_{1, j}(\mathbf{u})=1, \quad \varepsilon_{1, j}(\mathbf{u}), \eta_{1, j}(\mathbf{u}) \in O_{S}^{*},
$$

where $\varepsilon_{1, j}(\mathbf{u}), \eta_{1, j}(\mathbf{u})$ are the images of $\varepsilon_{1}, \eta_{1}$ under $\varphi_{\mathbf{u}, j}$.

We estimate from above the upper bound (6.3) from Proposition 6.1, By assumption, $f$ has degree at most $d_{1}$ and logarithmic height at most $h_{1}$, hence

$$
|f(\mathbf{u})| \leq d_{1}^{q} e^{h_{1}} \max (1,|\mathbf{u}|)^{d_{1}}=: R(\mathbf{u})
$$

Since the degree of $L$ is $d_{L} \leq D$, the cardinality $s$ of $S$ is at most $s \leq$ $D(1+\omega)$, where $\omega$ is the number of prime divisors of $f(\mathbf{u})$. Using the inequality from prime number theory, $\omega \leq O(\log |f(\mathbf{u})| / \log \log |f(\mathbf{u})|)$, we obtain

$$
s \leq O\left(\frac{D \log ^{*} R(\mathbf{u})}{\log ^{*} \log ^{*} R(\mathbf{u})}\right) .
$$

From this, one easily deduces that

$$
c_{1} \leq \exp O\left(D \log ^{*} R(\mathbf{u})\right) .
$$

Next, we estimate $P$ and $R_{S}$. By (6.5), we have

$$
P \leq Q \leq|f(\mathbf{u})|^{D} \leq \exp O\left(D \log ^{*} R(\mathbf{u})\right) .
$$

To estimate $R_{S}$, we use (6.1). By Lemma 5.5 (using $d_{0} \leq d_{1}$ ) we have

$$
\left|\Delta_{L}\right| \leq D^{2 D-1}\left(d_{1}^{q} e^{h_{1}} \max (1,|\mathbf{u}|)^{d_{1}}\right)^{2 D-2} \leq \exp O\left(D \log ^{*} R(\mathbf{u})\right),
$$

and this easily implies

$$
\left|\Delta_{L}\right|^{1 / 2}\left(\log ^{*} \Delta_{L}\right)^{D-1} \leq \exp O\left(D \log ^{*} R(\mathbf{u})\right) .
$$

Together with the estimates (6.6), (6.8) for $s$ and $Q$, this leads to

$$
R_{S} \leq \exp O\left(D \log ^{*} R(\mathbf{u})+s \log ^{*} \log ^{*} Q\right) \leq \exp O\left(D \log ^{*} R(\mathbf{u})\right) .
$$

Now by collecting (6.7)-(6.9), we infer that the right-hand side of (6.3) is bounded above by $\exp O\left(D \log ^{*} R(\mathbf{u})\right)$. So applying Proposition 6.1 to (6.4) gives

$$
h\left(\varepsilon_{1, j}(\mathbf{u})\right), h\left(\eta_{1, j}(\mathbf{u})\right) \leq \exp O\left(D \log ^{*} R(\mathbf{u})\right) .
$$


We apply Lemma 5.7 with $N:=4 D^{2}\left(q+d_{1}+1\right)^{2}$. From the already established (3.19) it follows that $\overline{\operatorname{deg}} \varepsilon_{1}, \overline{\operatorname{deg}} \eta_{1} \leq N$. Further, since $d_{1} \geq d_{0}$ we have $N \geq 2 D d_{0}+2\left(d_{1}+1\right)(q+1)$. So indeed, Lemma 5.7 is applicable with this value of $N$. It follows that the set $\mathcal{S}:=\left\{\mathbf{u} \in \mathbb{Z}^{q}:|\mathbf{u}| \leq N, \mathcal{H}(\mathbf{u}) \neq 0\right\}$ is not empty. Further, for $\mathbf{u} \in \mathcal{S}, j=1, \ldots, D$, we have

$$
\begin{aligned}
h\left(\varepsilon_{1, j}(\mathbf{u})\right) & \leq \exp O\left(D q \log d_{1}+D h_{1}+D d_{1} \log ^{*} N\right) \\
& \leq \exp O\left(N^{1 / 2} \log ^{*} N+D h_{1}\right),
\end{aligned}
$$

and so by Lemma 5.7 ,

$$
\bar{h}\left(\varepsilon_{1}\right) \leq \exp O\left(N^{1 / 2} \log ^{*} N+D h_{1}\right) .
$$

For $\bar{h}\left(\eta_{1}\right)$ we obtain the same upper bound. This easily implies (3.20) in the case $q>0$.

Now assume $q=0$. In this case, $K_{0}=\mathbb{Q}, A_{0}=\mathbb{Z}$ and $B=\mathbb{Z}\left[f^{-1}, y\right]$ where $y$ is an algebraic integer with minimal polynomial $\mathcal{F}=X^{D}+\mathcal{F}_{1} X^{D-1}+\cdots+$ $\mathcal{F}_{D} \in \mathbb{Z}[X]$ over $\mathbb{Q}$, and $f$ is a non-zero rational integer. By assumption, $\log |f| \leq h_{1}, \log \left|\mathcal{F}_{i}\right| \leq h_{1}$ for $i=1, \ldots, D$. Denote by $y_{1}, \ldots, y_{D}$ the conjugates of $y$, and let $L=\mathbb{Q}\left(y_{j}\right)$ for some $j$. By a similar argument as in the proof of Lemma 5.5, we have $\left|\Delta_{L}\right| \leq D^{2 D-1} e^{(2 D-2) h_{1}}$. The isomorphism given by $y \mapsto y_{j}$ maps $K$ to $L$ and $B$ to $O_{S}$, where $S$ consists of the infinite places of $L$ and of the prime ideals of $O_{L}$ that divide $f$. The estimates (6.5) - 6.9 ) remain valid if we replace $R(\mathbf{u})$ by $e^{h_{1}}$. Hence for any solution $\varepsilon_{1}, \eta_{1}$ of (3.18),

$$
h\left(\varepsilon_{1, j}\right), h\left(\eta_{1, j}\right) \leq \exp O\left(D h_{1}\right),
$$

where $\varepsilon_{1, j}, \eta_{1, j}$ are the $j$-th conjugates of $\varepsilon_{1}, \eta_{1}$, respectively. Now an application of Lemma 5.2 with $g=\mathcal{F}, m=D, \beta_{j}=\varepsilon_{1, j}$ gives

$$
\bar{h}\left(\varepsilon_{1}\right) \leq \exp O\left(D h_{1}\right) \text {. }
$$

Again we derive the same upper bound for $\bar{h}\left(\eta_{1}\right)$, and deduce (3.20). This completes the proof of Proposition 3.8.

\section{Proof of Theorem 1.3}

We start with some results on multiplicative (in)dependence.

Lemma 7.1. Let $L$ be an algebraic number field of degree $d$, and $\gamma_{0}, \ldots, \gamma_{s}$ non-zero elements of $L$ such that $\gamma_{0}, \ldots, \gamma_{s}$ are multiplicatively dependent, 
but any $s$ elements among $\gamma_{0}, \ldots, \gamma_{s}$ are multiplicatively independent. Then there are non-zero integers $k_{0}, \ldots, k_{s}$ such that

$$
\begin{aligned}
& \gamma_{0}^{k_{0}} \cdots \gamma_{s}^{k_{s}}=1 \\
& \left|k_{i}\right| \leq 58\left(s ! e^{s} / s^{s}\right) d^{s+1}(\log d) h\left(\gamma_{0}\right) \cdots h\left(\gamma_{s}\right) / h\left(\gamma_{i}\right) \text { for } i=0, \ldots, s .
\end{aligned}
$$

Proof. This is Corollary 3.2 of Loher and Masser [15, 2004]. They attribute this result to $\mathrm{Yu}$ Kunrui. Another result of this type was obtained earlier by Loxton and van der Poorten [17, 1983].

We prove a generalization for arbitrary finitely generated domains. As before, let $A=\mathbb{Z}\left[z_{1}, \ldots, z_{r}\right] \supseteq \mathbb{Z}$ be a domain, and suppose that the ideal $I$ of polynomials $f \in \mathbb{Z}\left[X_{1}, \ldots, X_{r}\right]$ with $f\left(z_{1}, \ldots, z_{r}\right)=0$ is generated by $f_{1}, \ldots, f_{m}$. Let $K$ be the quotient field of $A$. Let $\gamma_{0}, \ldots, \gamma_{s}$ be non-zero elements of $K$, and for $i=1, \ldots, s$, let $\left(g_{i 1}, g_{i 2}\right)$ be a pair of representatives for $\gamma_{i}$, i.e., elements of $\mathbb{Z}\left[X_{1}, \ldots, X_{r}\right]$ such that

$$
\gamma_{i}=\frac{g_{i 1}\left(z_{1}, \ldots, z_{r}\right)}{g_{i 2}\left(z_{1}, \ldots, z_{r}\right)} .
$$

Lemma 7.2. Assume that $\gamma_{0}, \ldots, \gamma_{s}$ are multiplicatively dependent. Further, assume that $f_{1}, \ldots, f_{m}$ and $g_{i 1}, g_{i 2}(i=0, \ldots, s)$ have degrees at most $d$ and logarithmic heights at most $h$, where $d \geq 1, h \geq 1$. Then there are integers $k_{0}, \ldots, k_{s}$, not all equal to 0 , such that

$$
\begin{aligned}
& \gamma_{0}^{k_{0}} \cdots \gamma_{s}^{k_{s}}=1 \\
& \left|k_{i}\right| \leq(2 d)^{\exp O(r+s)}(h+1)^{s} \text { for } i=0, \ldots, s .
\end{aligned}
$$

Proof. We assume without loss of generality that any $s$ numbers among $\gamma_{0}, \ldots, \gamma_{s}$ are multiplicatively independent (if this is not the case, take a minimal multiplicatively dependent subset of $\left\{\gamma_{0}, \ldots, \gamma_{s}\right\}$ and proceed further with this subset). We first assume that $q>0$. We use an argument of van der Poorten and Schlickewei [21, 1991]. We keep the notation and assumptions from Sections 3 5. In particular, we assume that $z_{1}, \ldots, z_{q}$ is a transcendence basis of $K$, and rename $z_{q+1}, \ldots, z_{r}$ as $y_{1}, \ldots, y_{t}$, respectively. For brevity, we have included the case $t=0$ as well in our proof. But it should be possible to prove in this case a sharper result by means of a more elementary method. In the case $t>0, y$ and $\mathcal{F}=X^{D}+\mathcal{F}_{1} X^{D-1}+\cdots+\mathcal{F}_{D}$ will be as in Corollary 3.4. In the case $t=0$ we take $m=1, f_{1}=0$, 
$d=h=1, y=1, \mathcal{F}=X-1, D=1$. We construct a specialization such that among the images of $\gamma_{0}, \ldots, \gamma_{s}$ no $s$ elements are multiplicatively dependent, and then apply Lemma 7.1 .

Let $V \geq 2 d$ be a positive integer. Later we shall make our choice of $V$ more precise. Let

$$
\begin{aligned}
\mathcal{V}:=\{\mathbf{v}= & \left(v_{0}, \ldots, v_{s}\right) \in \mathbb{Z}^{s+1} \backslash\{\mathbf{0}\}: \\
& \left.\left|v_{i}\right| \leq V \text { for } i=0, \ldots, s, \text { and with } v_{i}=0 \text { for some } i\right\} .
\end{aligned}
$$

Then

$$
\gamma_{\mathbf{v}}:=\left(\prod_{i=0}^{s} \gamma_{i}^{v_{i}}\right)-1 \quad(\mathbf{v} \in \mathcal{V})
$$

are non-zero elements of $K$. It is not difficult to show that for $\mathbf{v} \in \mathcal{V}, \gamma_{\mathbf{v}}$ has a pair of representatives $\left(g_{1, \mathbf{v}}, g_{2, \mathbf{v}}\right)$ such that

$$
\operatorname{deg} g_{1, \mathbf{v}}, \operatorname{deg} g_{2, \mathbf{v}} \leq s d V .
$$

In the case $t>0$, there exists by Lemma 3.6 a non-zero $f \in A_{0}$ such that

$$
A \subseteq B:=A_{0}\left[y, f^{-1}\right], \quad \gamma_{\mathbf{v}} \in B^{*} \text { for } \mathbf{v} \in \mathcal{V}
$$

and

$$
\operatorname{deg} f \leq V^{s+1}(2 s d V)^{\exp O(r)} \leq V^{\exp O(r+s)} .
$$

In the case $t=0$ this holds true as well, with $y=1$ and $f=\prod_{\mathbf{v} \in \mathcal{V}}\left(\left(g_{1, \mathbf{v}} \cdot g_{2, \mathbf{v}}\right)\right.$. We apply the theory on specializations explained in Section [5 with this $f$. We put $\mathcal{H}:=\Delta_{\mathcal{F}} \mathcal{F}_{D} f$, where $\Delta_{\mathcal{F}}$ is the discriminant of $\mathcal{F}$. Using Corollary 3.4 and inserting the bound $D \leq d^{t}$ from Lemma 3.2 we get for $t>0$,

$$
\left\{\begin{array}{l}
d_{0}:=\max \left(\operatorname{deg} f_{1}, \ldots, \operatorname{deg} f_{m}, \operatorname{deg} \mathcal{F}_{1}, \ldots, \operatorname{deg} \mathcal{F}_{D}\right) \leq(2 d)^{\exp O(r)} \\
h_{0}:=\max \left(h\left(f_{1}\right), \ldots, h\left(f_{m}\right), h\left(\mathcal{F}_{1}\right), \ldots, h\left(\mathcal{F}_{D}\right)\right) \leq(2 d)^{\exp O(r)}(h+1)
\end{array}\right.
$$

with the provision $\operatorname{deg} 0=h(0)=-\infty$ this is true also if $t=0$. Combining this with Lemma 3.5, we obtain

$$
\operatorname{deg} \mathcal{H} \leq(2 D-1) d_{0}+\operatorname{deg} f \leq V^{\exp O(r+s)} .
$$

By Lemma 5.3 there exists $\mathbf{u} \in \mathbb{Z}^{q}$ with

$$
\mathcal{H}(\mathbf{u}) \neq 0, \quad|\mathbf{u}| \leq V^{\exp O(r+s)} .
$$

We proceed further with this $\mathbf{u}$. 
As we have seen before, $\gamma_{\mathbf{v}} \in B^{*}$ for $\mathbf{v} \in \mathcal{V}$. By our choice of $\mathbf{u}$, there are $D$ distinct specialization maps $\varphi_{\mathbf{u}, j}(j=1, \ldots, D)$ from $B$ to $\overline{\mathbb{Q}}$. We fix one of these specializations, say $\varphi_{\mathbf{u}}$. Given $\alpha \in B$, we write $\alpha(\mathbf{u})$ for $\varphi_{\mathbf{u}}(\alpha)$. As the elements $\gamma_{\mathbf{v}}$ are all units in $B$, their images under $\varphi_{\mathbf{u}}$ are non-zero. So we have

$$
\prod_{i=0}^{s} \gamma_{i}(\mathbf{u})^{v_{i}} \neq 1 \text { for } \mathbf{v} \in \mathcal{V}
$$

where $\mathcal{V}$ is defined by $(\underline{7.3})$.

We use Lemma 5.6 to estimate the heights $h\left(\gamma_{i}(\mathbf{u})\right)$ for $i=0, \ldots, s$. Recall that by Lemma 3.5 we have

$$
\overline{\operatorname{deg}} \gamma_{i} \leq(2 d)^{\exp O(r)}, \quad \bar{h}\left(\gamma_{i}\right) \leq(2 d)^{\exp O(r)}(h+1)
$$

for $i=0, \ldots, s$. By inserting these bounds, together with the bound $D \leq d^{t}$ from Lemma 3.2, those for $d_{0}, h_{0}$ from (7.4) and that for $\mathbf{u}$ from (7.5) into the bound from Lemma 5.6, we obtain for $i=0, \ldots, s$,

$$
\begin{aligned}
h\left(\gamma_{i}(\mathbf{u})\right) & \leq(2 d)^{\exp O(r)}(1+h+\log \max (1,|\mathbf{u}|)) \\
& \leq(2 d)^{\exp O(r+s)}(1+h+\log V) .
\end{aligned}
$$

Assume that among $\gamma_{0}(\mathbf{u}), \ldots, \gamma_{s}(\mathbf{u})$ there are $s$ numbers which are multiplicatively dependent. By Lemma 7.1 there are integers $k_{0}, \ldots, k_{s}$, at least one of which is non-zero and at least one of which is 0 , such that

$$
\begin{aligned}
& \prod_{i=0}^{s} \gamma_{i}(\mathbf{u})^{k_{i}}=0, \\
& \left|k_{i}\right| \leq(2 d)^{\exp O(r+s)}(1+h+\log V)^{s-1} \text { for } i=0, \ldots, s .
\end{aligned}
$$

Now for

$$
V=(2 d)^{\exp O(r+s)}(h+1)^{s-1}
$$

(with a sufficiently large constant in the O-symbol), the upper bound for the numbers $\left|k_{i}\right|$ is smaller than $V$. But this would imply that $\prod_{i=0}^{s} \gamma_{i}(\mathbf{u})^{v_{i}}=1$ for some $\mathbf{v} \in \mathcal{V}$, contrary to (7.6). Thus we conclude that with the choice (17.8) for $V$, there exists $\mathbf{u} \in \mathbb{Z}^{q}$ with (7.5), such that any $s$ numbers among $\gamma_{0}(\mathbf{u}), \ldots, \gamma_{s}(\mathbf{u})$ are multiplicatively independent. Of course, the numbers $\gamma_{0}(\mathbf{u}), \ldots, \gamma_{s}(\mathbf{u})$ are multiplicatively dependent, since they are the images 
under $\varphi_{\mathbf{u}}$ of $\gamma_{0}, \ldots, \gamma_{s}$ which are multiplicatively dependent. Substituting (7.8) into (7.7) we obtain

$$
h\left(\gamma_{i}(\mathbf{u})\right) \leq(2 d)^{\exp O(r+s)}(h+1) \text { for } i=0, \ldots, s .
$$

Now Lemma 7.1 implies that there are non-zero integers $k_{0}, \ldots, k_{s}$ such that

$$
\begin{aligned}
& \prod_{i=0}^{s} \gamma_{i}(\mathbf{u})^{k_{i}}=1, \\
& \left|k_{i}\right| \leq(2 d)^{\exp O(r+s)}(h+1)^{s} \text { for } i=0, \ldots, s .
\end{aligned}
$$

Our assumption on $\gamma_{0}, \ldots, \gamma_{s}$ implies that there are non-zero integers $l_{0}, \ldots, l_{s}$ such that $\prod_{i=0}^{s} \gamma_{i}^{l_{i}}=1$. Hence $\prod_{i=0}^{s} \gamma_{i}(\mathbf{u})^{l_{i}}=1$. Together with (7.10) this implies

$$
\prod_{i=1}^{s} \gamma_{i}(\mathbf{u})^{l_{0} k_{i}-l_{i} k_{0}}=1 .
$$

But $\gamma_{1}(\mathbf{u}), \ldots, \gamma_{s}(\mathbf{u})$ are multiplicatively independent, hence $l_{0} k_{i}-l_{i} k_{0}=0$ for $i=1, \ldots, s$. That is,

$$
l_{0}\left(k_{0}, \ldots, k_{s}\right)=k_{0}\left(l_{0}, \ldots, l_{s}\right) .
$$

It follows that

$$
\prod_{i=0}^{s} \gamma_{i}^{k_{i}}=\rho
$$

for some root of unity $\rho$. But $\varphi_{\mathbf{u}}(\rho)=1$ and it is conjugate to $\rho$. Hence $\rho=1$. So in fact we have $\prod_{i=0}^{s} \gamma_{i}^{k_{i}}=1$ with non-zero integers $k_{i}$ satisfying (7.11). This proves our Lemma, but under the assumption $q>0$. If $q=$ 0 then a much simpler argument, without specializations, gives $h\left(\gamma_{i}\right) \leq$ $(2 d)^{\exp O(r+s)}(h+1)$ for $i=0, \ldots, s$ instead of (7.9) . Then the proof is finished in the same way as in the case $q>0$.

Corollary 7.3. Let $\gamma_{0}, \gamma_{1}, \ldots, \gamma_{s} \in K^{*}$, and suppose that $\gamma_{1}, \ldots, \gamma_{s}$ are multiplicatively independent and

$$
\gamma_{0}=\gamma_{1}^{k_{1}} \cdots \gamma_{s}^{k_{s}}
$$

for certain integers $k_{1}, \ldots, k_{s}$. Then

$$
\left|k_{i}\right| \leq(2 d)^{\exp O(r+s)}(h+1)^{s} \quad \text { for } i=1, \ldots, s .
$$


Proof. By Lemma 7.2, and by the multiplicative independence of $\gamma_{1}, \ldots, \gamma_{s}$, there are integers $l_{0}, \ldots, l_{m}$ such that

$$
\begin{aligned}
& \prod_{i=0}^{m} \gamma_{i}^{l_{i}}=1, \\
& l_{0} \neq 0, \quad\left|l_{i}\right| \leq(2 d)^{\exp O(r+s)}(h+1)^{s} \text { for } i=0, \ldots, s .
\end{aligned}
$$

Now clearly, we have also

$$
\prod_{i=1}^{s} \gamma_{i}^{l_{0} k_{i}-l_{i}}=1,
$$

hence $l_{0} k_{i}-l_{i}=0$ for $i=1, \ldots, s$. It follows that $\left|k_{i}\right|=\left|l_{i} / l_{0}\right| \leq$ $(2 d)^{\exp O(r+s)}(h+1)^{s}$ for $i=1, \ldots, s$. This implies our Corollary.

Proof of Theorem 1.3. We keep the notation and assumptions from the statement of Theorem 1.3. Define the domain

$$
\widetilde{A}:=A\left[\gamma_{1}, \gamma_{1}^{-1}, \ldots, \gamma_{s}, \gamma_{s}^{-1}\right]
$$

Then

$$
\widetilde{A} \cong \mathbb{Z}\left[X_{1}, \ldots, X_{r}, X_{r+1}, \ldots, X_{r+2 s}\right] / \widetilde{I}
$$

with

$$
\begin{array}{r}
\widetilde{I}=\left(f_{1}, \ldots, f_{m}, g_{12} X_{r+1}-g_{11}, g_{11} X_{r+2}-g_{12}, \ldots\right. \\
\left.\ldots, g_{s 2} X_{r+2 s-1}-g_{s 1}, g_{s 1} X_{r+2 s}-g_{s 2}\right) .
\end{array}
$$

Let $\left(v_{1}, \ldots, w_{s}\right)$ be a solution of (1.4), and put $\varepsilon:=\prod_{i=1}^{s} \gamma_{i}^{v_{i}}, \eta:=\prod_{i=1}^{s} \gamma_{i}^{w_{i}}$. Then

$$
a \varepsilon+b \eta=c, \quad \varepsilon, \eta \in \widetilde{A}^{*} .
$$

By Theorem 1.1, $\varepsilon$ has a representative $\widetilde{\varepsilon} \in \mathbb{Z}\left[X_{1}, \ldots, X_{r+2 s}\right]$ of degree and logarithmic height both bounded above by

$$
\exp \left((2 d)^{\exp O(r+s)}(h+1)\right) \text {. }
$$

Now Corollary 7.3 implies

$$
\left|v_{i}\right| \leq \exp \left((2 d)^{\exp O(r+s)}(h+1)\right) \text { for } i=1, \ldots, s .
$$

For $\left|w_{i}\right|(i=1, \ldots, s)$ we derive a similar upper bound. This completes the proof of Theorem 1.3 . 


\section{REFERENCES}

[1] M. Aschenbrenner, Ideal membership in polynomial rings over the integers, J. Amer. Math. Soc. 17 (2004), 407-442.

[2] A. BAKer, Contributions to the theory of Diophantine equations, Philos. Trans. Roy. Soc. London, Ser. A 263, 173-208.

[3] E. Bombieri, W. Gubler, Heights in Diophantine Geometry, Cambridge University Press, 2006.

[4] I. Borosh, M. Flahive, D. Rubin, B. Treybig, A sharp bound for solutions of linear Diophantine equations, Proc. Amer. Math. Soc. 105 (1989), 844-846.

[5] J. Contes, An effective p-adic analogue of a theorem of Thue, Acta Arith. 15 (1968/69), 279-305.

[6] K. GYŐRY, Sur les polynômes à coefficients entiers et de discriminant donné II, Publ. Math. Debrecen 21 (1974), 125-144.

[7] K. GYÖRY, On the number of solutions of linear equations in units of an algebraic number field, Comment. Math. Helv. 54 (1979), 583-600.

[8] K. GYőRY, Bounds for the solutions of norm form, discriminant form and index form equations in finitely generated domains, Acta Math. Hung. 42 (1983), 45-80.

[9] K. GYÖRY, Effective finiteness theorems for polynomials with given discriminant and integral elements with given discriminant over finitely generated domains, J. reine angew. Math. 346, 54-100.

[10] K. GYőRY, KunRui Yu, Bounds for the solutions of S-unit equations and decomposable form equations, Acta Arith. 123 (2006), 9-41.

[11] R. Hartshorne, Algebraic Geometry, Springer Verlag, 1977.

[12] G. Hermann, Die Frage der endlich vielen Schritte in der Theorie der Polynomideale, Math. Ann. 95 (1926), 736-788.

[13] S. LANG, Integral points on curves, Inst. Hautes Études Sci. Publ. Math. 6 (1960), $27-43$.

[14] D.J. Lewis, K. MahleR, On the representation of integers by binary forms, Acta Arith. 6 (1961), 333-363.

[15] T. Loher, D. Masser, Uniformly counting points of bounded height, Acta Arith. 111 (2004), 277-297.

[16] S. Louboutin, Explicit bounds for residues of dedekind zeta functions, values of L-functions at $s=1$, and relative class numbers, J. Number Theory 85 (2000), 263-282.

[17] J.H. Loxton, A.J. van Der Poorten, Multiplicative dependence in number fields, Acta Arith. 42 (1983), 291-302.

[18] K. MAhLER, Zur Approximation algebraischer Zahlen, I. (Über den größten Primteiler binärer Formen), Math. Ann. 107 (1933), 691-730.

[19] R.C. Mason, The hyperelliptic equation over function fields, Math. Proc. Camb. Philos. Soc. 93 (1983), 219-230.

[20] C.J. PARRY, The $\mathfrak{p}$-adic generalisation of the Thue-Siegel theorem, Acta Math. $\mathbf{8 3}$ (1950), 1-100. 
[21] A.J. van Der Poorten, H.P. Schlickewei, Additive relations in fields, J. Austral. Math. Soc. (Ser. A) 51 (1991), 154-170.

[22] P. Roquette, Einheiten und Divisorenklassen in endlich erzeugbaren Körpern, Jber. Deutsch. Math. Verein 60 (1958), 1-21.

[23] W.M. Schmidt, Thue's equation over function fields, J. Austral. Math. Soc. Ser. A 25 (1978), 385-422.

[24] A. Seidenberg, Constructions in algebra, Trans. Amer. Math. Soc. 197 (1974), 273-313.

[25] C.L. Siegel, Approximation algebraischer Zahlen, Math. Zeitschrift 10 (1921), 173213.

[26] H. Simmons, The solution of a decision problem for several classes of rings, Pacific J. Math. 34 (1970), 547-557.

J.-H. Evertse

Universiteit Leiden, Mathematisch Institudt,

Postbus 9512, 2300 RA Leiden, The Netherlands

E-mail address: evertse@math.leidenuniv.nl

K. GYőRY

Institute of Mathematics, University of Debrecen

Number Theory Research Group, Hungarian Academy of Sciences and

UNIVERSITY OF DEBRECEN

H-4010 Debrecen, P.O. Box 12, Hungary

E-mail address: gyory@science.unideb.hu 